\title{
Computing One-bit Compressive Sensing via Double-Sparsity Constrained Optimization
}

\author{
Shenglong Zhou, Ziyan Luo, Naihua Xiu, and Geoffrey Ye Li, Fellow, IEEE
}

\begin{abstract}
One-bit compressive sensing gains its popularity in signal processing and communications due to its low storage costs and low hardware complexity. However, it has been a challenging task to recover the signal only by exploiting the one-bit (the sign) information. In this paper, we appropriately formulate the one-bit compressive sensing into a double-sparsity constrained optimization problem. The first-order optimality conditions for this nonconvex and discontinuous problem are established via the newly introduced $\tau$-stationarity, based on which, a gradient projection subspace pursuit (GPSP) algorithm is developed. It is proven that GPSP can converge globally and terminate within finite steps. Numerical experiments have demonstrated its excellent performance in terms of a high order of accuracy with a fast computational speed.
\end{abstract}

Index Terms-One-bit compressive sensing, double-sparsity constrained optimization, optimality conditions, gradient projection subspace pursuit, global convergence

\section{INTRODUCTION}

$\mathbf{C}$ OMPRESSIVE sensing (CS) has seen evolutionary advances in theory and algorithms in the past few decades since introduced in the ground-breaking papers [1], [2], [3]. It aims to reconstruct a sparse signal $\mathrm{x}$ from an underdetermined linear system $\Phi \mathbf{x}=\mathbf{b}$, where $\Phi \in \mathbb{R}^{m \times n}$ is the measurement matrix and $\mathbf{b} \in \mathbb{R}^{m}$ is the measurement observation. To reduce storage costs and hardware complexity, in [4], only the sign information of measurements are preserved, that is

$$
\mathbf{c}=\operatorname{sgn}(\Phi \mathbf{x}) .
$$

Here, $\operatorname{sgn}(t)$ returns one if $t$ is positive and negative one otherwise, and thus $c_{i} \in\{1,-1\}, i \in[m]:=\{1,2, \cdots, m\}$ is the one-bit measurement. This gives rise to the one-bit CS. It was then extensively applied into applications including communications [5], [6], [7], [8], wireless sensor network [9], [10], [11], [12], cognitive radio [13], [14], imaging science [15], [16], [17] and to name a few. We refer to a couple of nice surveys [18], [19] for more applications.

\section{A. Related work}

a) Noiseless recovery. The task of one-bit CS constructs the sparse signal from the one-bit measurements. The ideal

This work is supported in part by the National Natural Science Foundation of China (12131004) and Beijing Natural Science Foundation (Z190002).

S.L. Zhou and G.Y. Li are with the ITP Lab, Department of Electrical and Electronic Engineering, Imperial College London, London SW72AZ, United Kingdom, e-mail: \{shenglong.zhou, geoffrey.li\}@imperial.ac.uk.

Z.Y. Luo and N.H. Xiu are with the Department of Applied Mathematics, Beijing Jiaotong University, Beijing 100044, People's Republic of China, email: \{zyluo,nhxiu\}@bjtu.edu.cn optimization model for recovery problem (1) is the following $l_{0}$-norm minimization,

$$
\min _{\mathbf{x} \in \mathbb{R}^{n}}\|\mathbf{x}\|_{0}, \quad \text { s.t. } \mathbf{c}=\operatorname{sgn}(\Phi \mathbf{x}),\|\mathbf{x}\|=1,
$$

where $\|\mathbf{x}\|_{0}$ counts the number of non-zero entries of $\mathbf{x}$ and $\|\mathbf{x}\|$ is its $l_{2}$-norm (Euclidean norm). We note that $\|\mathbf{x}\|_{0}$ is sometimes called $l_{0}$-norm, but it is actually not a norm in a strict mathematical sense. An impressive body of work has developed numerical algorithms for solving problem (2), but most of them focused on its approximations due to the NPhardness. The earliest work can be traced back to [4] where model (2) was relaxed by

$$
\min _{\mathbf{x} \in \mathbb{R}^{n}}\|\mathbf{x}\|_{1}, \quad \text { s.t. } A \mathbf{x} \geq 0,\|\mathbf{x}\|=1 .
$$

Here, $A:=\operatorname{Diag}(\mathbf{c}) \Phi, \operatorname{Diag}(\mathbf{c})$ represents the diagonal matrix with diagonal entries from $\mathbf{c}$, and $\|\cdot\|_{1}$ is the $l_{1}$-norm. Other relevant work on the case of exact recovery (1) includes the support recovery algorithm [20], the binary iterative reweighted method [21], the superset technique approximation [22], the fixed-point continuation algorithm [23], and modelbased deep learning [24].

b) Noisy recovery. In reality, measurement $\Phi \mathbf{x}$ is frequently contaminated by noise $\varepsilon$ before the quantization, i.e.,

$$
\mathbf{c}=\operatorname{sgn}(\Phi \mathbf{x}+\varepsilon) .
$$

For this scenario, a popular approach to reconstruct the signal benefits from the following optimization

$$
\min _{\mathbf{x} \in \mathbb{R}^{n}}\|\mathbf{x}\|_{1}+\lambda \varphi(\mathbf{x}), \quad \text { s.t. }\|\mathbf{x}\|=1,
$$

where $\lambda>0$ and $\varphi: \mathbb{R}^{n} \rightarrow \mathbb{R}$ is a loss function. In [4], they adopted the one-sided $l_{2}$ function $\varphi(\mathbf{x}):=\left\|(-A \mathbf{x})_{+}\right\|^{2}$ with $\mathbf{y}_{+}:=\left(\max \left\{y_{1}, 0\right\}, \cdots,\left\{y_{m}, 0\right\}\right)^{\top}$ and employed a renormalized fixed point iteration algorithm. Since the targeted problem is a nonconvex optimization, the convergence result has not been provided. The same problem was also addressed by a restricted step shrinkage algorithm [25], where the generated sequence was proved to converge to a stationary point of the penalty problem if some slightly strong assumptions on the sequence were satisfied.

Following the work in [4], Boufounos modified compressed sampling matching pursuit (CoSaMP) [26], one of the most popular greedy methods in CS, to derive the matching sign pursuit method [27]. It turned out to address the sparsity constrained model,

$$
\min _{\mathbf{x} \in \mathbb{R}^{n}} \varphi(\mathbf{x}), \quad \text { s.t. }\|\mathbf{x}\|_{0}=s,\|\mathbf{x}\|=1,
$$


where $s \ll n$ is a given sparsity level and $\varphi$ is the one-sided $l_{2}$ function. Based on the framework of the famous iterative hard thresholding algorithm, the modified version binary iterative hard thresholding (BIHT) was then developed in [28] to solve problem (6). Apart from the one-sided $l_{2}$ function, BITH was also able to process the one-sided $l_{1}$ function, namely, $\varphi(\mathbf{x})=\left\|(-A \mathbf{x})_{+}\right\|_{1}$. It was claimed that with a high probability, the distance between a reconstructed signal by BIHT and the original one can be bounded by a prefixed accuracy if the former quantizes to the same quantization point as the latter. As a consequence, the method enjoys a local convergence property. Recently, the normalized BIHT was investigated in [29], and achieves an approximation error rate optimal up to logarithmic factors with a high probability. Other work on noiseless recovery (4) consists of the convex relaxation [30], the passive algorithm [31], the soft consistency reconstructions [32], the pinball loss iterative hard thresholding [33], the sparse consistent coding algorithm [34], nonconvex penalty methods [35], and feature selection methods [36].

c) Noisy recovery with sign flips. When some signs are flipped after quantization, the scenario becomes

$$
\mathbf{c}=\operatorname{Diag}(\mathbf{h}) \operatorname{sgn}(\Phi \mathbf{x}+\varepsilon),
$$

where $h_{i} \in\{1,-1\}$ satisfying $\|\mathbf{h}-\mathbf{1}\|_{0} \leq k$ and $\varepsilon \in \mathbb{R}^{m}$ is the noise before quantization. Here, $k$ is a pre-estimated integer and can be deemed as the upper bound of the number of sign flips. Since there is an impressive body of work developing algorithms to address the above problem, see [37], [38], [39], we only review a small portion, which is enough to motivate our work in this paper.

In [40], authors took advantage of the $l_{1}$-regularized least squares and built the following model

$$
\min _{\mathbf{x} \in \mathbb{R}^{n}}\|\mathbf{x}\|_{1}+\lambda\|\mathbf{c}-\Phi \mathbf{x}\|^{2} .
$$

We note that this optimization problem does not model the loss of amplitude information in $\Phi \mathbf{x}$. However, as stated in [40], with a high probability, the distance between the solution to the model (up to a constant) and a sparse solution can be bounded by a prefixed accuracy if the sample size $m$ is greater than a threshold. Then a primal dual active set algorithm was proposed to solve the above model and proved to converge within one step under two assumptions: the submatrix of $\Phi$ indexed on the nonzero components of the sparse solution is full row rank and the initial point is sufficiently close to the sparse solution. Therefore, the generated sequence again has a local convergence property. Very recently, the authors in [41] replaced the $l_{1}$-norm in (8) by $\|\mathbf{x}\|_{p}^{p}:=\sum\left|x_{i}\right|^{p}(0<p<1)$ to design a weighted primal dual active set algorithm.

To exploit the information of sign flips (i.e., upper bound $k$ ), authors in [42] integrated a sparse variable $\mathbf{w}$ to problem (6). The nonzero components in $\mathbf{w}$ represent the measurements that have sign flips. The resulting optimization problem is

$$
\begin{array}{cl}
\min _{\mathbf{x} \in \mathbb{R}^{n}, \mathbf{w} \in \mathbb{R}^{m}} & \left\|(-\operatorname{Diag}(\mathbf{c})(\Phi \mathbf{x}+\mathbf{w}))_{+}\right\|_{p}^{p}, \\
\text { s.t. } & \|\mathbf{x}\|_{0} \leq s,\|\mathbf{x}\|=1,\|\mathbf{w}\|_{0} \leq k,
\end{array}
$$

where $p=1$ or 2 . To tackle the above problem, an alternating minimization method (adaptive outliers pursuit, AOP) was cast: solving one variable while fixing the other. However, AOP has been tested to heavily rely on the choice of $k$ and the convergence result remains to be seen. Other work relating to (9) includes the noise-adaptive renormalized fixed point iteration approach [43] and the noise-adaptive restricted step shrinkage [44].

When upper bound $k$ of the number of sign flips is unavailable, a remedy pursues a solution with sign flips as few as possible, which can be fulfilled by the following one-sided $l_{0}$ function minimization [45],

$$
\min _{\mathbf{x} \in \mathbb{R}^{n}}\left\|(\epsilon \mathbf{1}-A \mathbf{x})_{+}\right\|_{0}+\eta\|\mathbf{x}\|^{2} \text {, s.t. }\|\mathbf{x}\|_{0} \leq s,
$$

where $\eta$ and $\epsilon$ are given positive parameters. Here, $\epsilon$ is used to majorize the objective function. The first term in the objective function arises from maximizing a posterior estimation from the perspective of statistics. It returns the number of positive components of $(\epsilon \mathbf{1}-A \mathbf{x})$ and can be regarded as the number of the sign flips when $\epsilon$ is quite small. Instead of solving the one-sided $l_{0}$ model directly, a fixed-point algorithm [45] was created for its approximation,

$$
\begin{array}{ll}
\min _{\mathbf{x} \in \mathbb{R}^{n}, \mathbf{w} \in \mathbb{R}^{m}} & \left\|(\epsilon \mathbf{1}-\mathbf{w})_{+}\right\|_{0}+\mu\|\mathbf{w}-A \mathbf{x}\|^{2}+\eta\|\mathbf{x}\|^{2}, \\
\text { s.t. } & \|\mathbf{x}\|_{0} \leq s,
\end{array}
$$

where $\mu>0$. It has shown that the generated sequence converges to a local minimizer of the approximation problem if the spectral norm of matrix $A$ is bounded by some chosen parameters. However, the relationship between the solution obtained by the method and the one to the original problem (10) has not been well explored.

To summarize, all these aforementioned methods either had no convergence guarantees (e.g., [4], [27], [42], [43]) or had convergence results that required more or less assumptions on data $\Phi$ or $A$ (e.g., [40], [45]). A natural question is whether there is a proper model based on which the proposed algorithm can converge without any assumptions. Moreover, empirical numerical experiments have demonstrated that algorithms that only exploit information of sparsity did not render desirable accuracies for the case of noisy recovery with sign flips (7). By contrast, as shown by numerical experiments in [42], [43], [44], [45], the recovery accuracies have been improved greatly when the information of sign flips was taken into consideration.

\section{B. Our contributions}

To eliminate the assumptions on data $\Phi$ or $A$ for better convergence results, we need a loss function with more pleasant features. Moreover, to enhance the recovery accuracy, it is suggested by the work in [42], [45] that we should take full advantage of the information of both sparsity and the number of sign flips. Based on these two aspects, we formulate one-bit CS problem (7) as the following double-sparsity constrained optimization:

$$
\begin{array}{cl}
\min _{\mathbf{x} \in \mathbb{R}^{n}, \mathbf{y} \in \mathbb{R}^{m}} & \|A \mathbf{x}+\mathbf{y}-\epsilon \mathbf{1}\|^{2}+\eta\|\mathbf{x}\|^{2}, \\
\text { s.t. } & \|\mathbf{x}\|_{0} \leq s, \quad\left\|\mathbf{y}_{+}\right\|_{0} \leq k,
\end{array}
$$

where $\eta>0$ is a penalty parameter, $s \ll n$ and $k \ll m$ are two integers representing the prior information on the upper 
bounds of the signal sparsity and the number of sign flips, respectively. When penalizing the sign flip constraint in our model, it turns to (11) with $\mathbf{y}=\epsilon \mathbf{1}-\mathbf{w}$.

Now we would like to emphasize the merits of our proposed optimization model (12). For starters, it is able to deal with scenarios (1), (4), and (7). For the first two cases without sign flips, we just set $k=0$ in model (12). Moreover, it has a strongly convex quadratic objective function, which is beneficial to develop a fast second-order algorithm and establish its convergence results without any additional assumptions, see Theorems 5.1 and 5.2. Finally, the new model exploits both the sparsity and the upper bound of the number of sign flips, thereby yielding a high order of accuracy, as shown in Figure 6. It is worth mentioning that the selection of $k$ is very flexible (see Figure 5), which reveals that our approach does not rely on $k$ heavily (while AOP in [42] does).

The main contributions in this paper are threefold:

i. The new optimization model. The double-sparsity constrained optimization, problem (12), is formulated to handle the one-bit CS. It is well-known that the two discrete and nonconvex constraints in (12) lead to the NP-hardness in general. Nevertheless, a necessary and sufficient optimality condition as stated in (21) for a local minimizer is established, see Lemma 3.1. Moreover, the necessary or sufficient optimality condition for a global minimizer is further studied through the newly introduced $\tau$-stationary point, see Theorem 3.1. Finally, it turns out that the distance between any local minimizer to (12) (up to a constant) and the true signal can be bounded by an error bound with a high probability under some assumptions, see Theorem 4.1.

ii. The efficient GP SP algorithm. As the established optimality conditions indicating a $\tau$-stationary point is instructive to pursue an optimal solution to (12), we design a gradient projection method with a subspace pursuit scheme interpolated (dubbed as GPSP). The proposed method is proved to be globally convergent to a $\tau$ stationary point (denoted by $\mathbf{z}^{*}$ ), which must be a unique local minimizer of (12) without any assumptions. Moreover, if we assume an additional condition, then $\mathbf{z}^{*}$ can be a global minimizer, as shown in Theorem 5.1. Furthermore, the produced sequence is eventually identical to $\mathbf{z}^{*}$, namely, GPSP can stop within finite steps, see Theorem 5.2.

iii. High numerical performance. GP SP is demonstrated to be relatively robust to parameters $k, \epsilon, \eta$ in (12) in the numerical experiments, which indicates that we do not need an exact upper bound $k$ of the sign flips. In addition, GP SP is cast based on problem (12) and turns out to be a second-order method, thereby leading to a considerably high order of accuracy. Finally, the algorithmic design enables it to have a low computational complexity. Therefore, it outperforms all benchmark solvers for synthetic data, in terms of time efficiency and recovery accuracy.

\section{Organization}

The remainder of the paper is organized as follows. In Section II, some necessary mathematical backgrounds are provided, including the notation and the projection onto the feasible set of problem (12). Section III is devoted to the optimality conditions of the problem, associated with the $\tau$ stationary points, followed by its relationship to the global minimizers. In Section IV, we investigate the reconstruction performance to the recovery accuracy of solutions to (12) and the true signal. In Section $\mathrm{V}$, the gradient projection subspace pursuit (GP SP) method is designed, and properties of the global convergence and termination within finite steps are established. Numerical experiments are given in Section VI, including the involved parameters tuning and comparisons with other six excellent solvers. Concluding remarks are made in Section VII.

\section{PRELIMINARIES}

We first define some notation employed throughout this paper. To differ from $\operatorname{sgn}(\mathrm{t})$, the sign function is written as $\operatorname{sign}(\mathrm{t})$ that returns 0 if $t=0$ and $\operatorname{sgn}(\mathrm{t})$ otherwise. Given a subset $T \subseteq[n]:=\{1,2, \cdots, n\}$, its cardinality and complementary set are $|T|$ and $\bar{T}:=[n] \backslash T$. For a vector $\mathbf{x} \in \mathbb{R}^{n}$, the support set, $\operatorname{supp}(\mathbf{x})$, represents the indices of nonzero elements of $\mathbf{x}$ and the neighbourhood with a radius $\delta>0$ is denoted by $N(\mathbf{x}, \delta):=\left\{\mathbf{w} \in \mathbb{R}^{n}:\|\mathbf{w}-\mathbf{x}\|<\delta\right\}$. Let $\|\cdot\|_{\infty}$ be the infinity norm, $\|\mathbf{x}\|_{[i]}$ be the $i$ th largest (in absolute) element of $\mathbf{x}$, and $\|A\|_{2}$ be the spectral norm of $A$. In addition, $\mathbf{x}_{T}$ stands for the sub-vector contains elements of $\mathbf{x}$ indexed on $T$. Similarly, for a matrix $A \in \mathbb{R}^{m \times n}, A_{\Gamma T}$ is the sub-matrix containing rows indexed on $\Gamma$ and columns indexed on $T$, particularly, $A_{: T}=A_{[m] T}$. Moreover, we merge two vectors $\mathbf{x}$ and $\mathbf{y}$ by $\mathbf{z}:=(\mathbf{x} ; \mathbf{y}):=\left(\mathbf{x}^{\top} \mathbf{y}^{\top}\right)^{\top}$. For a positive definite matrix $H$, the $H$-weighted norm is written $\|\mathbf{z}\|_{H}^{2}=\langle\mathbf{z}, H \mathbf{z}\rangle$, where $\left\langle\mathbf{z}, \mathbf{z}^{\prime}\right\rangle:=\sum z_{i} z_{i}^{\prime}$ is the inner product of two vectors. Given a scalar $a \in \mathbb{R},\lceil a\rceil$ returns the smallest integer that is no less than $a$. For simplicity, denote

$$
\begin{aligned}
& S:=\left\{\mathbf{x} \in \mathbb{R}^{n}:\|\mathbf{x}\|_{0} \leq s\right\}, \\
& K:=\left\{\mathbf{y} \in \mathbb{R}^{m}:\left\|\mathbf{y}_{+}\right\|_{0} \leq k\right\} .
\end{aligned}
$$

The feasible region of (12) is then denoted by

$$
\mathcal{F}:=S \times K
$$

\section{A. Projections}

For a nonempty and closed set $\Omega \subseteq \mathbb{R}^{n}$, the projection $\Pi_{\Omega}(\mathbf{x})$ of $\mathbf{x} \in \mathbb{R}^{n}$ onto $\Omega$ is given by

$$
\Pi_{\Omega}(\mathbf{x})=\operatorname{argmin}\{\|\mathbf{x}-\mathbf{w}\|: \mathbf{w} \in \Omega\} .
$$

By introducing

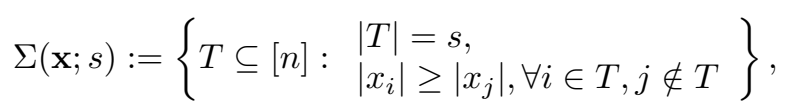

one can easily verify that

$$
\Pi_{S}(\mathbf{x})=\left\{\left(\mathbf{x}_{T} ; 0\right): T \in \Sigma(\mathbf{x} ; s)\right\} .
$$

To derive the projection of a point $\mathbf{y} \in \mathbb{R}^{m}$ onto $K$, denote

$$
\begin{aligned}
\Gamma_{+} & :=\left\{i \in[m]: y_{i}>0\right\}, \\
\Gamma_{0} & :=\left\{i \in[m]: y_{i}=0\right\}, \\
\Gamma_{-} & :=\left\{i \in[m]: y_{i}<0\right\} .
\end{aligned}
$$


Note that $\Gamma_{+}, \Gamma_{0}$ and $\Gamma_{-}$should depend on $\mathbf{y}$. We drop their dependence if no extra explanations are provided for the sake of notational convenience. Based on the above notation, for a point $\mathbf{y} \in \mathbb{R}^{m}$ and an integer $k \in[m]$, we define a set by

$$
\begin{aligned}
\Theta(\mathbf{y} ; k) & \left\{\begin{array}{l}
\Gamma_{k} \subseteq \Gamma_{+},\left|\Gamma_{k}\right|=\min \left\{k,\left|\Gamma_{+}\right|\right\} \\
\Gamma_{k} \cup \Gamma_{-}: \\
y_{i} \geq y_{j} \geq 0, \forall i \in \Gamma_{k}, \forall j \in \Gamma_{+} \backslash \Gamma_{k}
\end{array}\right\},
\end{aligned}
$$

where $\Gamma_{+}$and $\Gamma_{-}$are given by (16). One can observe that $\Gamma \in$ $\Theta(\mathbf{y} ; k)$ consists of the indices of all negative elements and the first $\min \left\{k,\left|\Gamma_{+}\right|\right\}$largest positive elements of $\mathbf{y}$. These notation allow us to derive projection $\Pi_{K}(\mathbf{y})$ by

$$
\Pi_{K}(\mathbf{y})=\left\{\left(\mathbf{y}_{\Gamma} ; 0\right): \Gamma \in \Theta(\mathbf{y} ; k)\right\} .
$$

For example, $\mathbf{y}=(3,2,2,0,-2)^{\top}$, then we have

$$
\begin{aligned}
\Theta(\mathbf{y} ; 3) & =\{\{1,2,3,5\}\}, \Pi_{K}(\mathbf{y})=\{\mathbf{y}\}, \\
\Theta(\mathbf{y} ; 2) & =\{\{1,2,5\},\{1,3,5\}\}, \\
\Pi_{K}(\mathbf{y}) & =\left\{(3,2,0,0,-2)^{\top},(3,0,2,0,-2)^{\top}\right\} .
\end{aligned}
$$

\section{B. Properties of the objective function}

To end this section, we present some properties of the objective function in (12), which can be written as follows

$$
\begin{aligned}
f(\mathbf{x}, \mathbf{y}) & :=\|A \mathbf{x}+\mathbf{y}-\epsilon \mathbf{1}\|^{2}+\eta\|\mathbf{x}\|^{2} \\
& =\|\mathbf{z}\|_{H}^{2}-2 \epsilon\left\langle\left(A^{\top} \mathbf{1} ; \mathbf{1}\right), \mathbf{z}\right\rangle+m \epsilon^{2} \\
& =: \quad f(\mathbf{z}),
\end{aligned}
$$

where $H$ is given by

$$
H:=\frac{1}{2} \nabla^{2} f(\mathbf{z})=\left[\begin{array}{ll}
A^{\top} A+\eta I & A^{\top} \\
A & I
\end{array}\right] .
$$

It is easy to verify that $H$ is symmetric positive definite and hence has all eigenvalues positive. Denote the smallest and the largest eigenvalues by $\lambda_{\min }$ and $\lambda_{\max }$, respectively. The quadratic objective function, $f$, is then strongly convex and strongly smooth since for any $\mathbf{z}$ and $\mathbf{z}^{\prime}$ in $\mathbb{R}^{n+m}$,

$$
\begin{aligned}
& f(\mathbf{z})-f\left(\mathbf{z}^{\prime}\right)-\left\langle\nabla f\left(\mathbf{z}^{\prime}\right), \mathbf{z}-\mathbf{z}^{\prime}\right\rangle \\
= & \left\|\mathbf{z}-\mathbf{z}^{\prime}\right\|_{H}^{2} \in\left[\lambda_{\min }\left\|\mathbf{z}-\mathbf{z}^{\prime}\right\|^{2}, \lambda_{\max }\left\|\mathbf{z}-\mathbf{z}^{\prime}\right\|^{2}\right] .
\end{aligned}
$$

\section{Optimality CONDitions}

The first-order necessary and sufficient optimality conditions for problem (12) are established in this section and all proofs are given in Appendix A.

Lemma 3.1: Consider a point $\mathbf{z}^{*}:=\left(\mathbf{x}^{*} ; \mathbf{y}^{*}\right) \in \mathcal{F}$ with

$$
T^{*}:=\operatorname{supp}\left(\mathbf{x}^{*}\right), \quad \Gamma^{*}:=\operatorname{supp}\left(\mathbf{y}^{*}\right) .
$$

A point $\mathbf{z}^{*}$ is a local minimizer of (12) if and only if it satisfies

$$
\begin{aligned}
& \nabla_{\mathbf{x}} f\left(\mathbf{z}^{*}\right)=0, \quad \text { if } \quad\left\|\mathbf{x}^{*}\right\|_{0}<s, \\
& \left(\nabla_{\mathbf{x}} f\left(\mathbf{z}^{*}\right)\right)_{T^{*}}=0 \text {, if } \quad\left\|\mathbf{x}^{*}\right\|_{0}=s \text {, } \\
& \left(\nabla_{\mathbf{y}} f\left(\mathbf{z}^{*}\right)\right)_{\Gamma^{*}}=0, \quad \text { and }\left\|\mathbf{y}_{+}^{*}\right\|_{0}=k \text {. }
\end{aligned}
$$

Furthermore, for any local minimizer $\mathbf{z}^{*}$, there is a $\delta_{*}>0$ satisfying the following quadratic growth property

$$
f(\mathbf{z})-f\left(\mathbf{z}^{*}\right) \geq\left\|\mathbf{z}-\mathbf{z}^{*}\right\|_{H}^{2}, \quad \forall \mathbf{z} \in \mathcal{F} \cap N\left(\mathbf{z}^{*}, \delta_{*}\right) .
$$

Remark 3.1: If $\mathrm{x}^{*}=0$ is the optimal solution to problem (12), then $\mathbf{y}^{*}=\left(\mathbf{y}_{\Gamma^{*}}^{*} ; 0\right)=(\epsilon \mathbf{1} ; 0)$ for any $\left|\Gamma^{*}\right|=k$. From Lemma 3.1, $\nabla_{\mathbf{x}} f\left(\mathbf{z}^{*}\right)=-2 \epsilon A_{\bar{\Gamma}^{*}}^{\top} \mathbf{1}=0$. Therefore, if we need to exclude zero solution, we assume $\left\|A_{\Upsilon:}^{\top} \mathbf{1}\right\|_{\infty}>0$ for any $|\Upsilon|=m-k$. This is a very weak assumption.

Lemma 3.1 shows the optimality conditions of a point being a local minimizer. We further establish the conditions for a global minimizer. To do that, we introduce a $\tau$-stationary point. A point $\mathbf{z}^{*}:=\left(\mathbf{x}^{*} ; \mathbf{y}^{*}\right)$ is called a $\tau$-stationary point of (12) with some $\tau>0$ if it satisfies

$$
\mathbf{z}^{*} \in \Pi_{\mathcal{F}}\left(\mathbf{z}^{*}-\tau \nabla f\left(\mathbf{z}^{*}\right)\right) .
$$

An equivalent characterization is presented as follows.

Lemma 3.2: A point $\mathbf{z}^{*}$ is a $\tau$-stationary point of problem (12) with some $\tau>0$ if and only if it satisfies

$$
\begin{aligned}
& \left\|\mathbf{x}^{*}\right\|_{0} \leq s, \\
& \tau\left(\nabla_{\mathbf{x}} f\left(\mathbf{z}^{*}\right)\right)_{i} \begin{cases}=0, & i \in T^{*}, \\
\in\left[-\left\|\mathbf{x}^{*}\right\|_{[s]},\left\|\mathbf{x}^{*}\right\|_{[s]}\right], & i \in \bar{T}^{*},\end{cases} \\
& \left\|\mathbf{y}_{+}^{*}\right\|_{0}=k, \\
& \tau\left(\nabla_{\mathbf{y}} f\left(\mathbf{z}^{*}\right)\right)_{i} \begin{cases}=0, & i \in \Gamma^{*}, \\
\in\left[-\left\|\mathbf{y}_{+}^{*}\right\|_{[k]}, 0\right], & i \in \bar{\Gamma}^{*} .\end{cases}
\end{aligned}
$$

The following theorem reveals the relationships between $\tau$ stationary points and global minimizers of problem (12).

Theorem 3.1: For problem (12) and a point $\mathbf{z}^{*} \in \mathcal{F}$, a global minimizer $\mathbf{z}^{*}$ is a $\tau$-stationary point with $0<\tau \leq 1 /\left(2 \lambda_{\max }\right)$, and conversely, a $\tau$-stationary point with $\tau \geq 1 /\left(2 \lambda_{\min }\right)$ is also a global minimizer.

Based on the above theorem, we can check if a local minimizer is a global minimizer explicitly with the help of the $\tau$-stationary point.

Corollary 3.1: Let $\mathbf{z}^{*}$ be a local minimizer of problem (12). Then it is a $\tau_{*}$-stationary point with

$$
\tau_{*}:= \begin{cases}\frac{\left\|\mathbf{y}_{+}^{*}\right\|_{[k]}}{\left\|\boldsymbol{\xi}^{*}\right\|_{\infty}}, & \left\|\mathbf{x}^{*}\right\|_{0}<s, \\ \min \left\{\frac{\left\|\mathbf{y}_{+}^{*}\right\|_{[k]}}{\left\|\boldsymbol{\xi}^{*}\right\|_{\infty}}, \frac{\left\|\mathbf{x}^{*}\right\|_{[s]}}{\left\|A_{\bar{\Gamma}^{*} \bar{T}^{*}}^{\top} \boldsymbol{\xi}^{*}\right\|_{\infty}}\right\}, & \left\|\mathbf{x}^{*}\right\|_{0}=s,\end{cases}
$$

where $\boldsymbol{\xi}^{*}:=2 \epsilon \eta\left(A_{\bar{\Gamma}^{*} T^{*}} A_{\bar{\Gamma}^{*} T^{*}}^{\top}+\eta I\right)^{-1} \mathbf{1}$. Moreover, $\mathbf{z}^{*}$ is also a global minimizer of problem (12) if $\tau_{*} \geq 1 /\left(2 \lambda_{\min }\right)$.

We give a simple example to illustrate the above corollary.

Example 3.1: Consider problem (12) with $s=1, k=1$ and $A \in \mathbb{R}^{3 \times 4}$ given by

$$
A:=\left[\begin{array}{rrrr}
-1 & \mathrm{t} & \mathrm{t} & 0 \\
1 & \mathrm{t} & 0 & 0 \\
1 & 0 & \mathrm{t} & \mathrm{t}
\end{array}\right]
$$

where $t>0$. Let $c:=\frac{2 \epsilon}{2+\eta}$. Now for point $\mathbf{z}^{*}=\left(\mathbf{x}^{*} ; \mathbf{y}^{*}\right)$ with $\mathbf{x}^{*}=(c, 0,0,0)^{\top}$ and $\mathbf{y}^{*}=(c+\epsilon, 0,0)^{\top}$, one can check that $\nabla_{\mathbf{y}} f\left(\mathbf{z}^{*}\right)=-c \eta(0,1,1)^{\top}$ and $\nabla_{\mathbf{x}} f\left(\mathbf{z}^{*}\right)=-t c \eta(0,1,1,1)^{\top}$. Therefore, $\left(\mathbf{x}^{*}, \mathbf{y}^{*}\right)$ is a local minimizer of problem (12) since it satisfies (21). Moreover, direct calculations can check that

$$
\boldsymbol{\xi}^{*}=c \eta \mathbf{1} \text { and } \tau_{*}=\min \left\{\frac{4+\eta}{2+\eta}, \frac{1}{\eta t}\right\} .
$$


We plot $1 /\left(2 \lambda_{\min }\right)$ and $\tau_{*}$ by fixing $\eta=1$ or fixing $t=1 / 5$ in Figure 1 . One can see that $\tau_{*}>1 /\left(2 \lambda_{\min }\right)$ when $t \in(0,0.4]$ in Figure $1 \mathrm{a}$ or when $\eta \in[0.1,6]$ in Figure 1b, which by Corollary 3.1 means that $\mathbf{z}^{*}$ is a global minimizer of problem (12). This example indicates that there are many cases of $A$ and $\eta$ for which the found local minimizer is global.

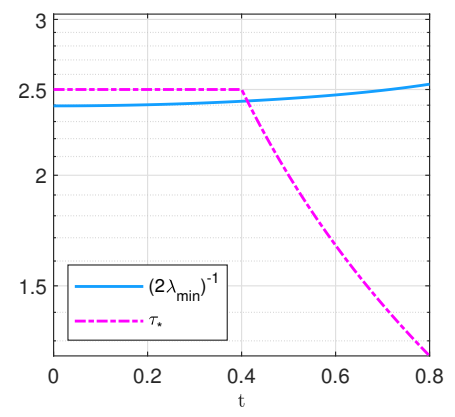

(a) Fixing $\eta=1$.

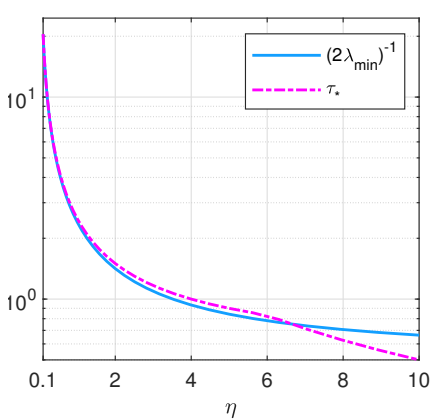

(b) Fixing $t=1 / 5$.
Fig. 1: Values of $1 /\left(2 \lambda_{\min }\right)$ and $\tau_{*}$.

\section{Reconstruction Performance}

In this section, we investigate the reconstruction performance of our proposed optimization model (12). Hereafter, we denote the ground-truth signal by $\mathrm{x}^{\text {true }}$ with

$$
\left\|\mathbf{x}^{\text {true }}\right\|_{0} \leq s, \quad\left\|\mathbf{x}^{\text {true }}\right\|=1
$$

Moreover, let $\mathbf{z}^{*}=\left(\mathbf{x}^{*} ; \mathbf{y}^{*}\right)$ with $\mathbf{x}^{*} \neq 0$ be any local minimizer of problem (12) and

$$
\widehat{\mathbf{x}}:=\mathrm{x}^{*} /\left\|\mathrm{x}^{*}\right\| \text {. }
$$

To exclude zero solution $\mathbf{x}^{*}=0$, as shown in Remark 3.1, we assume $\left\|A_{\Upsilon:}^{\top} \mathbf{1}\right\|_{\infty}>0$ for any $|\Upsilon|=m-k$ in this section. We now aim at estimating the bound for the gap between $\widehat{x}$ and $\mathrm{x}^{\text {true }}$. To proceed with that, we also need the concept of the binary $\delta$-stable embedding of a mapping [28, Definition 1]. Let $\delta \in(0,1)$, a mapping $\mathcal{A}: \mathbb{R}^{n} \rightarrow\{-1,1\}^{m}$ is a binary $\delta$-stable embedding $(\mathrm{B} \delta \mathrm{SE})$ of order $s$ for sparse vectors if

$$
d(\mathbf{u}, \mathbf{v})-\delta \leq \frac{1}{m}\|\mathcal{A}(\mathbf{u})-\mathcal{A}(\mathbf{v})\|_{0} \leq d(\mathbf{u}, \mathbf{v})+\delta,
$$

for all $\mathbf{u}, \mathbf{v}$ satisfying $|\operatorname{supp}(\mathbf{u}) \cup \operatorname{supp}(\mathbf{v})| \leq s$ and $\|\mathbf{u}\|=$ $\|\mathbf{v}\|=1$, where

$$
d(\mathbf{u}, \mathbf{v}):=\frac{1}{\pi} \arccos \langle\mathbf{u}, \mathbf{v}\rangle
$$

The following result states that mapping $\operatorname{sgn}(\cdot)$ is a $\mathrm{B} \delta \mathrm{SE}$.

Lemma 4.1 ([28, Theorem 3]): Let $\delta \in(0,1), \Phi$ be a matrix with entries generated from the independent and identically distributed (i.i.d.) samples of the standard Gaussian distribution $\mathcal{N}(0,1)$ and the number of measurements satisfies

$$
m \geq m(s, \delta, \theta):=\frac{2}{\delta^{2}}(s \log (n)+2 s \log (35 / \delta)+\log (2 / \theta))
$$

for a fixed $\theta \in[0,1]$, then

$$
\mathbb{P}\left\{\left|d(\mathbf{u}, \mathbf{v})-\|\mathcal{A}(\mathbf{u})-\mathcal{A}(\mathbf{v})\|_{0} / m\right| \leq \delta\right\} \geq 1-\theta .
$$

Lemma 4.2: Let $\mathbf{z}^{*}$ be any local minimizer of problem (12) and $c_{A}$ represent the minimal eigenvalue of $A_{\bar{\Gamma} T} A_{\bar{\Gamma} T}^{\top}$ for any $|\Gamma| \geq k$ and $|T| \leq s$. Then for any

$$
0 \leq \eta \leq \frac{c_{A}}{\sqrt{m-k}-1},
$$

point $\widehat{\mathrm{x}}$ satisfies

$$
\|\widehat{\mathbf{x}}\|=1, \quad\|\widehat{\mathbf{x}}\|_{0} \leq s, \quad\left\|(-A \widehat{\mathbf{x}})_{+}\right\|_{0} \leq k .
$$

Theorem 4.1: Let $\varepsilon$ be a noise vector with entries from i.i.d. samples of $\mathcal{N}\left(0, \varrho^{2}\right)$ and $\Phi$ be generated as Lemma 4.1 with $m \geq m(2 s, \delta, \theta)$ for a fixed $\theta \in[0,1]$ and $\delta \in(0,1)$ satisfying

$$
\phi:=\frac{k}{m}+\frac{\varrho}{4}+\delta \in(0,1 / 2] .
$$

Let $\mathbf{z}^{*}$ be any local minimizer of problem (12) with $\eta$ being chosen as (29), then

$$
\mathbb{P}\left\{\left\|\widehat{\mathbf{x}}-\mathbf{x}^{\text {true }}\right\| \leq 2 \sin (\phi \pi)\right\} \geq 1-e^{-2 m \delta^{2}}-\theta .
$$

Remark 4.1: We have some comments on Theorem 4.1.

- The result is valid for any local minimizer. If $\phi$ in (31) tends to zero, then we can conclude that any local minimizer approaches the true signal with a high probability, which indicates there only exists one local minimizer of problem (12). This local minimizer is also the unique global minimizer and is the true signal.

- The reconstruction performance apparently depends on sparsity level $s$, upper bound $k$ as well as number of measurements $m$. It also provides a hint to set the regularized parameter, $\eta$, by (29), though the calculation of $c_{A}$ is quite expensive. Nevertheless, (29) means that $\eta$ should be chosen smaller than a threshold in practical computation. In other words, it should not be set too large, which can be verified by our numerical experiments, see Figure 2. Moreover, the establishments of Theorem 4.1 and Lemma 4.2 do not impose assumptions on parameter $\epsilon$. Therefore, the reconstruction performance might be quite robust to $\epsilon$. This is well testified by our numerical simulation, see Figure 3.

\section{Gradient Projection Subspace Pursuit}

A gradient projection method with a subspace pursuit strategy is proposed to handle problem (12) by seeking a $\tau$-stationary point. For notational simplicity, hereafter, for a parameter $\tau \in(0,1]$, we always let

$$
\mathbf{z}^{\ell}(\tau)=\left[\mathbf{x}^{\ell}(\tau) ; \mathbf{y}^{\ell}(\tau)\right] \in \Pi_{\mathcal{F}}\left(\mathbf{z}^{\ell}-\tau \nabla f\left(\mathbf{z}^{\ell}\right)\right),
$$

for the $\ell$ th iteration $\mathbf{z}^{\ell}:=\left(\mathbf{x}^{\ell} ; \mathbf{y}^{\ell}\right)$. Analogous to the $\Gamma$-related indices defined for $\mathbf{y}$ in (16), we also define

$$
\begin{aligned}
& \widetilde{\Gamma}_{+}^{\ell}:=\left\{i \in[m]:\left(\mathbf{y}^{\ell}\left(\tau_{\ell}\right)\right)_{i}>0\right\} \\
& \Gamma_{+}^{\ell}:=\left\{i \in[m]: y_{i}^{\ell}>0\right\} \\
& \Gamma_{0}^{\ell}:=\left\{i \in[m]: y_{i}^{\ell}=0\right\} \\
& \Gamma_{-}^{\ell}:=\left\{i \in[m]: y_{i}^{\ell}<0\right\} .
\end{aligned}
$$

We denote the support sets for $\mathbf{x}^{\ell}$ and $\mathbf{x}^{\ell}\left(\tau_{\ell}\right)$ as follows

$$
T^{\ell}:=\operatorname{supp}\left(\mathbf{x}^{\ell}\right), \quad \widetilde{T}^{\ell}:=\operatorname{supp}\left(\mathbf{x}^{\ell}\left(\tau_{\ell}\right)\right) .
$$


Given $\mathbf{z}^{\ell}=\left(\mathbf{x}^{\ell} ; \mathbf{y}^{\ell}\right) \in \mathcal{F}$, define the following subspace

$$
\Omega\left(\mathbf{z}^{\ell}\right):=\left\{\mathbf{z}=(\mathbf{x} ; \mathbf{y}): \begin{array}{l}
\mathbf{x}_{\bar{T}^{\ell}}=0, \\
\mathbf{y}_{\Gamma_{0}^{\ell}}=0, \mathbf{y}_{\Gamma_{-}^{\ell}} \leq 0
\end{array}\right\} .
$$

It is easy to see that $\Omega\left(\mathbf{z}^{\ell}\right) \subseteq \mathcal{F}$. Based on these notation, we summarize the framework of the proposed method in Algorithm 1.

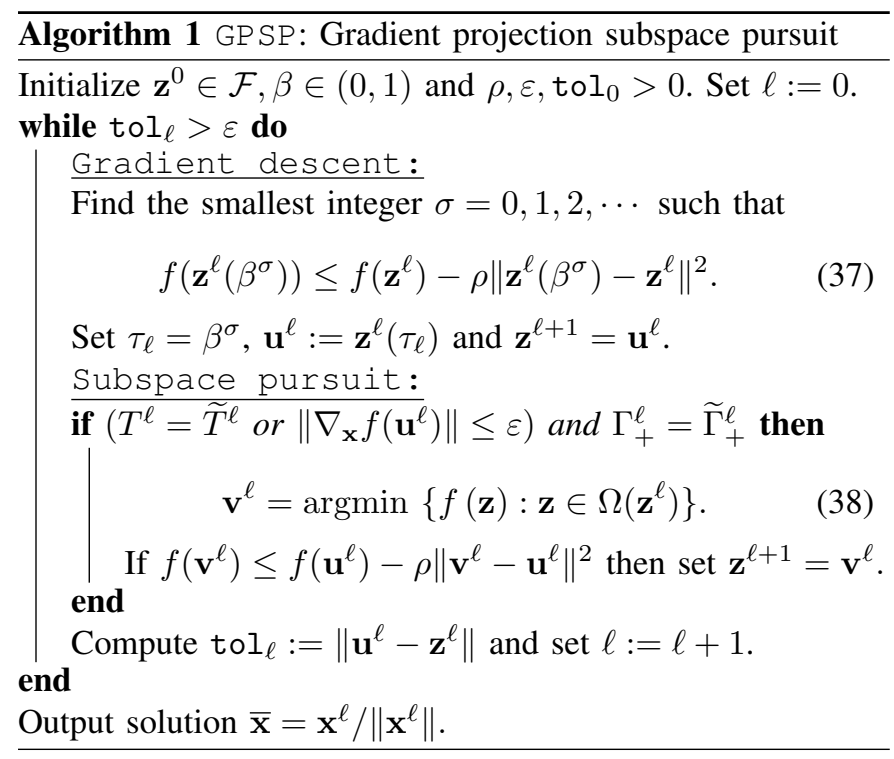

Observing that initial point $\mathbf{z}^{0} \in \mathcal{F}$, and $\Omega\left(\mathbf{z}^{\ell}\right) \subseteq \mathcal{F}$, we can see that all iterations are feasible. Particularly, if gap tol $l_{\ell}=$ $\left\|\mathbf{z}^{\ell}-\mathbf{u}^{\ell}\right\|$ vanishes, then

$$
\mathbf{z}^{\ell}=\mathbf{u}^{\ell} \in \Pi_{\mathcal{F}}\left(\mathbf{z}^{\ell}-\tau_{\ell} \nabla f\left(\mathbf{z}^{\ell}\right)\right),
$$

which indicates that $\mathbf{z}^{\ell}$ is a $\tau$-stationary point with $\tau \leq \tau_{\ell}$. Additionally, once conditions $T^{\ell}=\widetilde{T}^{\ell}$ and $\Gamma_{+}^{\ell}=\widetilde{\Gamma}_{+}^{\ell}$ are satisfied, we have $\mathbf{u}^{l} \in \Omega\left(\mathbf{z}^{\ell}\right)$. The unique minimizer, $\mathbf{v}^{\ell}$, of $f$ over $\Omega\left(\mathbf{z}^{\ell}\right)$ implies that

$$
\left\langle\nabla f\left(\mathbf{v}^{\ell}\right), \mathbf{u}^{\ell}-\mathbf{v}^{\ell}\right\rangle \geq 0 .
$$

In virtue of (20), we have

$$
f\left(\mathbf{v}^{\ell}\right) \leq f\left(\mathbf{u}^{\ell}\right)-\lambda_{\min }\left\|\mathbf{u}^{\ell}-\mathbf{v}^{\ell}\right\|^{2} .
$$

Suppose $0<\rho \leq \lambda_{\min }$. Then candidate $\mathbf{v}^{\ell}$ will be taken, namely, $\mathbf{z}^{\ell+1}=\mathbf{v}^{\ell}$.

\section{A. Computational complexity analysis}

To update $\mathbf{u}^{\ell}$, we need to select one point from $\Pi_{\mathcal{F}}\left(\mathbf{z}^{\ell}-\right.$ $\left.\tau \nabla f\left(\mathbf{z}^{\ell}\right)\right)$. Namely, three quantities are computed: $\overline{\mathbf{z}}^{\ell}:=$ $\left(\overline{\mathbf{x}}^{\ell} ; \overline{\mathbf{y}}^{\ell}\right):=\mathbf{z}^{\ell}-\tau \nabla f\left(\mathbf{z}^{\ell}\right), \Pi_{S}\left(\overline{\mathbf{x}}^{\ell}\right)$ and $\Pi_{K}\left(\overline{\mathbf{y}}^{\ell}\right)$. For the former, the computational complexity is about $O(m n)$. To select one point from $\Pi_{S}\left(\overline{\mathbf{x}}^{\ell}\right)$, we only pick the first $s$ largest (in absolute) elements of $\overline{\mathbf{x}}^{\ell}$. This allows us to use a MATLAB built-in function maxk whose computational complexity is $O(n+s \log s)$. Similarly, for $\Pi_{K}\left(\overline{\mathbf{y}}^{\ell}\right)$, the computational complexity is $O(m+k \log k)$. Thus, updating $\mathbf{u}^{\ell}$ takes a computational complexity of order $O(\sigma m n)$, where $\sigma$ is the smallest integer satisfying (37).
To update $\mathbf{v}^{\ell}$, we solve a quadratic programming,

$$
\begin{aligned}
\mathbf{v}^{\ell}=\underset{(\mathbf{x} ; \mathbf{y})}{\operatorname{argmin}} & \|A \mathbf{x}+\mathbf{y}-\epsilon \mathbf{1}\|^{2}+\eta\|\mathbf{x}\|^{2} \\
\text { s.t. } & \mathbf{x}_{\bar{T}^{\ell}}=0, \mathbf{y}_{\Gamma_{0}^{\ell}}=0, \mathbf{y}_{\Gamma_{-}^{\ell}} \leq 0,
\end{aligned}
$$

for fixed $T^{\ell}, \Gamma_{0}^{\ell}$ and $\Gamma_{-}^{\ell}$. Any solvers for solving the quadratic programming can be used to solve (41) to pursue a solution in good quality. To further reduce the computation cost, we drop constraint $\mathbf{y}_{\Gamma^{\ell}} \leq 0$ from (41) and simply solve the equations:

$$
\left[\begin{array}{cc}
A_{: T^{\ell}}^{\top} A_{: T^{\ell}}+\eta I & A_{\bar{\Gamma}_{0}^{\ell} T^{\ell}}^{\top} \\
A_{\bar{\Gamma}_{0}^{\ell} T^{\ell}} & I
\end{array}\right]\left[\begin{array}{c}
\mathbf{x}_{T^{\ell}} \\
\mathbf{y}_{\bar{\Gamma}_{0}^{\ell}}
\end{array}\right]=\left[\begin{array}{r}
A_{: T^{\ell}}^{\top} \epsilon \mathbf{1} \\
\\
\end{array}\right] .
$$

The solution, $\left(\widetilde{\mathbf{x}}^{\ell} ; \widetilde{\mathbf{y}}^{\ell}\right)$, can be derived by

$$
\begin{aligned}
\widetilde{\mathbf{x}}_{T^{\ell}}^{\ell} & =\left[A_{\Gamma_{0}^{\ell} T^{\ell}}^{\top} A_{\Gamma_{0}^{\ell} T^{\ell}}+\eta I\right]^{-1}\left[A_{\Gamma_{0}^{\ell} T^{\ell}}^{\top} \in \mathbf{1}\right], \\
\widetilde{\mathbf{x}}_{T^{\ell}}^{\ell} & =0, \\
\widetilde{\mathbf{y}}_{\Gamma_{0}^{\ell}}^{\ell} & =0, \\
\widetilde{\mathbf{y}}_{\bar{\Gamma}_{0}^{\ell}}^{\ell} & =\epsilon \mathbf{1}-A_{\bar{\Gamma}_{0}^{\ell} T^{\ell}} \widetilde{\mathbf{x}}_{T^{\ell}}^{\ell} .
\end{aligned}
$$

If $\widetilde{\mathbf{y}}_{\Gamma^{\ell}}^{\ell} \leq 0$, namely, $\left(\widetilde{\mathbf{x}}^{\ell} ; \widetilde{\mathbf{y}}^{\ell}\right)$ is the solution to (41), then we set $\mathbf{v}^{\ell}=\left(\widetilde{\mathbf{x}}^{\ell} ; \widetilde{\mathbf{y}}^{\ell}\right)$. Otherwise, this point will not be taken into consideration, and we set $\mathbf{z}^{\ell+1}=\mathbf{u}^{\ell}$. The computational complexity of addressing the above equations is about $O\left(m s^{2}+s^{3}\right)$.

Overall, the computational complexity of each iteration is

$$
O\left(\sigma m n+m s^{2}+s^{3}\right) .
$$

\section{B. Convergence analysis}

The first result shows that Armijo-type step size (see [46] for more details) $\left\{\tau_{\ell}\right\}$ is well defined.

Lemma 5.1: For any $0<\tau \leq \frac{1}{2 \rho+2 \lambda_{\max }}$, it holds that

$$
f\left(\mathbf{z}^{\ell}(\tau)\right) \leq f\left(\mathbf{z}^{\ell}\right)-\rho\left\|\mathbf{z}^{\ell}(\tau)-\mathbf{z}^{\ell}\right\|^{2},
$$

and thus $\inf _{\ell \geq 0}\left\{\tau_{\ell}\right\} \geq \underline{\tau}>0$, where

$$
\underline{\tau}:=\min \left\{1, \frac{\beta}{2 \rho+2 \lambda_{\max }}\right\} .
$$

Lemma 5.2: Let $\left\{\mathbf{z}^{\ell}\right\}$ be the sequence generated by GP SP and $\tau$ be given by (43). Then the following results hold.

i) Sequence $\left\{\mathbf{z}^{\ell}\right\}$ is bounded and $\lim _{\ell \rightarrow \infty}\left\|\mathbf{z}^{\ell+1}-\mathbf{z}^{\ell}\right\|=$ $\lim _{\ell \rightarrow \infty}\left\|\mathbf{u}^{\ell}-\mathbf{z}^{\ell}\right\|=0$.

ii) Any accumulating point of $\left\{\mathbf{z}^{\ell}\right\}$ is a $\tau$-stationary point with $0<\tau \leq \underline{\tau}$ of problem (12).

The above lemma allows us to conclude that the whole sequence converges to a unique local minimizer without any assumptions. But with an additional condition, the whole sequence can achieve a global minimizer.

Theorem 5.1: The whole sequence, $\left\{\mathbf{z}^{\ell}\right\}$, generated by GPSP converges to a $\tau$-stationary point $\mathbf{z}^{*}$ of problem (12), which is necessarily a unique local minimizer. If $\mathbf{z}^{*}$ is also a $\tau_{*}$-stationary point with $\tau_{*} \geq 1 /\left(2 \lambda_{\min }\right)$, where $\tau_{*}$ is defined by (25), then it is a global minimizer.

The following theorem claims that GP SP can terminate at the limit of the sequence after a certain point. 
Theorem 5.2: Let $\left\{\mathbf{z}^{\ell}\right\}$ be the sequence generated by GP SP with $0<\rho \leq \lambda_{\min }$ and $\mathbf{z}^{*}$ be its limiting point. Then GPSP will terminate at $\mathbf{z}^{*}$ within finite steps, namely, there is a finite $\kappa \geq 1$ such that

$$
\mathbf{z}^{\ell}=\mathbf{v}^{\kappa}=\mathbf{z}^{*}, \quad \forall \ell>\kappa .
$$

Finally, we can even show that the distance between every iterate $\mathrm{x}^{\ell}$ and true signal $\mathrm{x}^{\text {true }}$ has an upper bound.

Theorem 5.3: Let $\Phi$ and $\varepsilon$ be given as in Theorem 4.1, $\left\{\mathbf{z}^{\ell}\right\}$ be the sequence generated by GPSP and $\mathbf{z}^{*}$ be its limiting point. We choose $\eta$ as (29) and $\rho$ as $0<\rho \leq \lambda_{\min }$. Then there is a finite $\kappa \geq 1$ such that, for any $\ell \geq \kappa$,

$$
\mathbb{P}\left\{\left\|c_{*} \mathbf{x}^{\ell}-\mathbf{x}^{\text {true }}\right\| \leq 2 \sin (\phi \pi)\right\} \geq 1-e^{-2 m \delta^{2}}-\theta .
$$

\section{Comparisons with other methods}

We would like to compare GPSP with some other methods that have been proposed to deal with the noisy recovery with sign flips, namely, model (7). Their comparisons on the theoretical guarantees and the computational complexity are summarized in Table I. Only three methods OSLO, PDASC and GPSP have been established convergence results. Since PDASC converges only when the initial point is chosen close to the accumulating point of the sequence, it has a local convergence property. Both OSLO and PDASC also require some assumptions on data $\Phi$ or $A$ to derive the convergence. Moreover, we note that WPDASC, PDASC and GPSP are the second-order methods since they make use of Hessian matrix (i.e., the second-order information) of the objective function. Therefore, they have slightly higher computational complexity. Here, $s_{\ell}$ is the sparsity level of the point at $\ell$ th iteration. However, empirical numerical experiments have demonstrated that the second-order methods can converge within much fewer steps and yield a much higher order of accuracy than the firstorder methods. This is another reason that GP SP is capable of delivering relatively desirable accuracy.

TABLE I: Comparisons of different algorithms.

\begin{tabular}{lccr}
\hline Algs. & Convergence & $\begin{array}{c}\text { Assumptions } \\
\text { on } \Phi \text { or } A\end{array}$ & Complexity \\
\hline \multicolumn{5}{c}{ First-order methods } \\
\hline RBIHT [37] & -- & -- & $O(m n)$ \\
AOPF [42] & -- & -- & $O(m n)$ \\
NARSS [44] & -- & -- & $O(m n)$ \\
OSL0 [45] & Global & Yes & $O(m n)$ \\
\hline \multicolumn{5}{c}{ Second-order methods } \\
\hline WPDASC [41] & -- & -- & $O\left(m n+m s_{\ell}^{2}+s_{\ell}^{3}\right)$ \\
PDASC [40] & Local & Yes & $O\left(m n+m s_{\ell}^{2}+s_{\ell}^{3}\right)$ \\
GPSP & Global & No & $O\left(\sigma m n+m s^{2}+s^{3}\right)$ \\
\hline
\end{tabular}

Remark 5.1: We now highlight the difference between GP SP and OSLO in [45] developed to solve (11), which is a penalized version our proposed model (12). Firstly, the different frameworks mean that GP SP is a second-order method while OSLO is a first-order method. Moreover, OSLO converges to a local minimizer if the spectral norm of matrix $A$ is bounded by some chosen parameters and converges to a global minimizer if further assuming that the initial point is sufficiently close to this global minimizer. However, GP SP converges to a unique local minimizer without any assumptions on $A$ and converges to a global minimizer if $\tau_{*} \geq 1 /\left(2 \lambda_{\min }\right)$. Hence it does not impose any condition on the initial point.

\section{NUMERICAL EXPERIMENTS}

In this section, we will conduct extensive numerical experiments to showcase the performance of our proposed GP SP (available at https://github.com/ShenglongZhou/GPSP), by using MATLAB (R2019a) on a laptop of 32GB memory and Inter(R) Core(TM) i9-9880H 2.3Ghz CPU.

\section{A. Testing examples}

Examples with the data generated from the Gaussian distributions are taken into account.

Example 6.1 (Independent covariance [42], [45]): Entries of $\Phi:=\left[\phi_{1}, \cdots, \phi_{m}\right]^{\top} \in \mathbb{R}^{m \times n}$ and the nonzero entries of ground-truth $s_{*}$-sparse vector $\mathbf{x}^{\text {true }} \in \mathbb{R}^{n}$ (i.e., $\left.\left\|\mathbf{x}^{\text {true }}\right\|_{0} \leq s_{*}\right)$ are generated from the independent and identically distributed (i.i.d.) samples of the standard Gaussian distribution, $\mathcal{N}(0,1)$. To avoid tiny nonzero entries of $\mathbf{x}^{\text {true }}$, let $x_{i}^{\text {true }}=x_{i}^{\text {true }}+\operatorname{sign}\left(x_{i}^{\text {true }}\right)$ for nonzero $x_{i}^{\text {true }}$, followed by normalizing $\mathbf{x}^{\text {true }}$ to be a unit vector. Let $\mathbf{c}^{\text {true }}=\operatorname{sgn}\left(\Phi \mathbf{x}^{\text {true }}\right)$ and $\mathbf{c}=\operatorname{Diag}(\mathbf{h}) \operatorname{sgn}\left(\Phi \mathbf{x}^{\text {true }}+\boldsymbol{\varepsilon}\right)$, where entries of noise $\varepsilon$ are the i.i.d. samples of $\mathcal{N}\left(0,0.1^{2}\right)$ and $\lceil\mathrm{rm}\rceil$ entries of $\mathbf{h}$ are randomly selected to be -1 , where $r$ is the flipping ratio.

Example 6.2 (Correlated covariance [40]): Rows of $\Phi$ are generated from the i.i.d. samples of $\mathcal{N}(0, \Sigma)$ with $\Sigma_{i j}=$ $v^{|i-j|}, i, j \in[n]$, where $v \in(0,1)$. Then $\mathbf{x}^{\text {true }}, \mathbf{c}^{\text {true }}$, and $\mathbf{c}$ are generated the same as those in Example 6.1.

To demonstrate the performance of one method, apart from the CPU TIME, we will also report the signal-to-noise ratio (SNR) in $\mathrm{dB}$, the Hamming error $(\mathrm{HE})$ and the Hamming distance (HD). They are defined by

$$
\begin{aligned}
\mathrm{SNR} & :=-20 \log _{10}\left\|\mathbf{x}-\mathbf{x}^{\text {true }}\right\|, \\
\mathrm{HD} & :=(1 / m)\|\operatorname{sgn}(\Phi \mathbf{x})-\mathbf{c}\|_{0}, \\
\mathrm{HE} & :=(1 / m)\left\|\operatorname{sgn}(\Phi \mathbf{x})-\mathbf{c}^{\text {true }}\right\|_{0},
\end{aligned}
$$

where $\mathbf{x}$ is the solution obtained by one method. The larger SNR (or the smaller HE or HD) means the better recovery.

\section{B. Implementation and parameter selection}

We terminate GPSP if $\left\|\mathbf{z}^{\ell}-\mathbf{u}^{\ell}\right\| \leq \varepsilon$ or $\ell>2000$ and set $\beta=0.5, \rho=10^{-6}, \varepsilon=10^{-4}$ and $\mathbf{z}^{0}=0$ in all experiments. Parameters $\epsilon, \eta, s$ and $k$ in (12) are tuned as follows.

(i) Selection of $\eta$. Although Theorem 4.1 suggested that $\eta$ should be set as (29), it is difficult to estimate the value of the right hand side of (29). Therefore, we tested a wide range of $\eta$ (e.g, $\eta \in\left[0,10^{4}\right]$ ) to see how it effects our algorithm. To proceed with that, for Example 6.1, we fix $\left(n, s_{*}, r, \epsilon\right)=(500,5,0.05,0.01)$ and $k=\lceil 0.01 m\rceil$ but vary $m \in\{0.25,0.5,0.75,1\} n$ and $\eta \in\left\{0,10^{-8}, 10^{-7}, \cdots, 10^{4}\right\}$. Average results over 200 trials are reported in Figure 2. It can be evidently seen that results are stabilized when $\eta \in[0,10]$ while getting worse when $\eta>10$ is rising. Similar trends 
are also observed for GPSP solving Example 6.2. This well testifies that $\eta$ should be chosen smaller than a threshold, as shown in (29). Therefore, any value in $[0,10]$ can be used to set $\eta$. For simplicity, we fix $\eta=10^{-4}$.
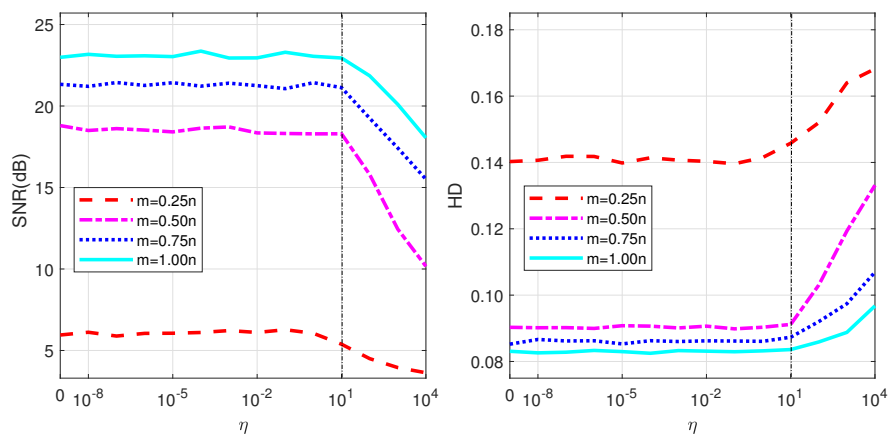

Fig. 2: Effect of $\eta$ for Example 6.1.

(ii) Selection of $\epsilon$. As mentioned in Remark 4.1, GP SP might be quite robust to the choices of $\epsilon$ since the error bound theory (i.e., Theorem 4.1) does not impose any assumptions on $\epsilon$. To testify that, we fix $\left(n, s_{*}, r\right)=(500,5,0.05)$ and $k=\lceil 0.01 m\rceil$ but alter $m \in\{0.25,0.5,0.75,1\} n$ and $\epsilon \in\left[10^{-10}, 10^{10}\right]$ for GP SP solving Example 6.1. As presented in Figure 3, the average results over 200 trials do not fluctuate significantly when varying $\epsilon$, which shows robustness of GP SP to the choices of $\epsilon$ in range $\left[10^{-10}, 10^{10}\right]$. An underlying explanation is as follows: In our numerical experiments, we observed that $\left\|\mathrm{x}^{\ell}\right\|$ was increasing along with the rising of $\epsilon$. So the final iteration, $\mathbf{x}^{\ell}$, is indeed influenced by $\epsilon$. However, after the normalization, namely, $\bar{x}=\mathbf{x}^{\ell} /\left\|\mathbf{x}^{\ell}\right\|$, the impact of $\epsilon$ is eliminated. Since similar performance can be seen for GP SP solving Example 6.2, the corresponding results are omitted. For simplicity, we fix $\epsilon=0.01$ in the subsequent numerical experiments.
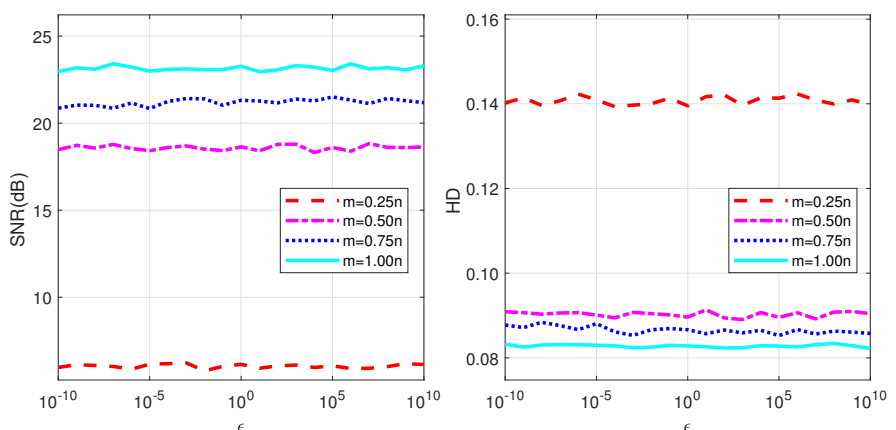

Fig. 3: Effect of $\epsilon$ for Example 6.1.

(iii) Selection of $s$. Sparsity level $s$ clearly has a heavy influence of the recovery quality. As shown in Figure 4 where $\left(n, m, s_{*}, r\right)=(500,250,5,0.05)$, the ground-truth signal, $\mathrm{x}^{\text {true }}$, has $s_{*}=5$ nonzero components with their indices denoted by $T^{\text {true }}=\operatorname{supp}\left(\mathbf{x}^{\text {true }}\right)$. Apparently, GP SP gets the most accurate signal if we set $s=s_{*}$ because it almost exactly recovers those nonzero components. For $s=s_{*}-2=3$, the recovered signal has 3 nonzero components, however, their indices belong to the true support set, $T^{\text {true }}$. While for $s=s_{*}+2$ or $s=s_{*}+4$, GP SP generates a solution $\mathbf{x}$ whose support set $\operatorname{supp}(\mathbf{x})$ covers $T^{\text {true }}$ with extra incorrect indices. However, compared with magnitude $\left|x_{i}\right|, i \in T^{\text {true }}$, those redundant nonzero components $\left|x_{i}\right|, i \in \operatorname{supp}(\mathbf{x}) \backslash T^{\text {true }}$ are pretty small. If we remove those small parts and normalize the signal to have a unit length, then the new signal is much closer to $\mathbf{x}^{\text {true }}$. For simplicity, we set $s=s_{*}$ in the sequel.
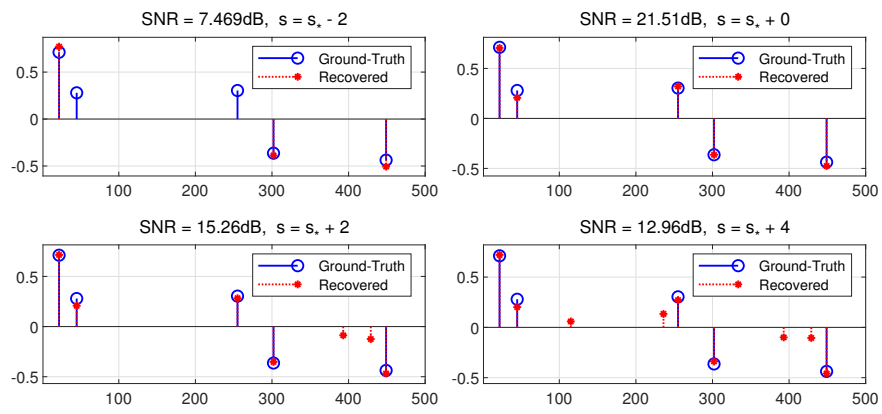

Fig. 4: Effect of $s$ for Example 6.1.

(iv) Selection of $k$. Note that $k$ is the upper bound of the number of sign flips of $\Phi \mathbf{x}$ and is usually unknown beforehand. However, model (12) does not require an exact $k$. One could either fix it by a small integer (e.g. $k=\lceil 0.01 \mathrm{~m}\rceil$ ) or start with a slightly bigger value and reduce it iteratively. We tested GPSP for solving Example 6.1 and Example 6.2 under both schemes and corresponding numerical performance does not have a big difference. For instance, as indicated in Figure 5, where $\left(n, m, s_{*}, v\right)=(500,250,5,0.5)$, we select $k / m \in\{0.01,0.03,0.05,0.07,0.09\}$ and then fix it for GP SP. Evidently, for each case of flipping ratio $r$, results SNR and HD do not vary significantly along with $k$ altering.

We note that in the above numerical experiments, the upper bound, $k$, was unequal to true number of sign flips $\mathrm{rm}$ but close to $\mathrm{rm}$. However, we have also tested much larger $\mathrm{k} / \mathrm{m}$ (e.g., 0.2) than $r$ (e.g., 0.02), the recovery accuracies were degraded greatly. Therefore, we could conclude that GPSP is quite robust to these $k$ around the true number of sign flips, which suggests that $k$ should not be chosen too far away from $\mathrm{rm}$. Hence, in our numerical experiments, we pick $k=\lceil 0.01 \mathrm{~m}\rceil$ if no additional information is provided.

\section{Benchmark methods}

Six state-of-the-art solvers are selected for comparisons. They are BIHT [28], AOPF (BIHT-AOP-flip, [42]), PAOPF (PIHT-AOP-flip, [33]), PIHT (This code was implemented based on the pinball loss from [33] to address the noisy recovery with sign flips), PDASC [40] and WPDASC [41]. We note that BIHT behaves well for noiseless recovery but has shown undesirable performance for noisy recovery. We include it in comparison since we use its obtained solution $\mathbf{x}$ to generate the upper bound of the number of sign flips for AOPF, PAOPF, and PIHT, namely, set $L=\|\operatorname{sgn}(\Phi \mathbf{x})-\mathbf{c}\|_{0}$. This is one option to generate the upper bound suggested by [42]. Like our method, the first four methods need to specify sparsity level $s$ that is set by $s=s_{*}$. Moreover, to accelerate the termination of PIHT, we stop it when the number of 

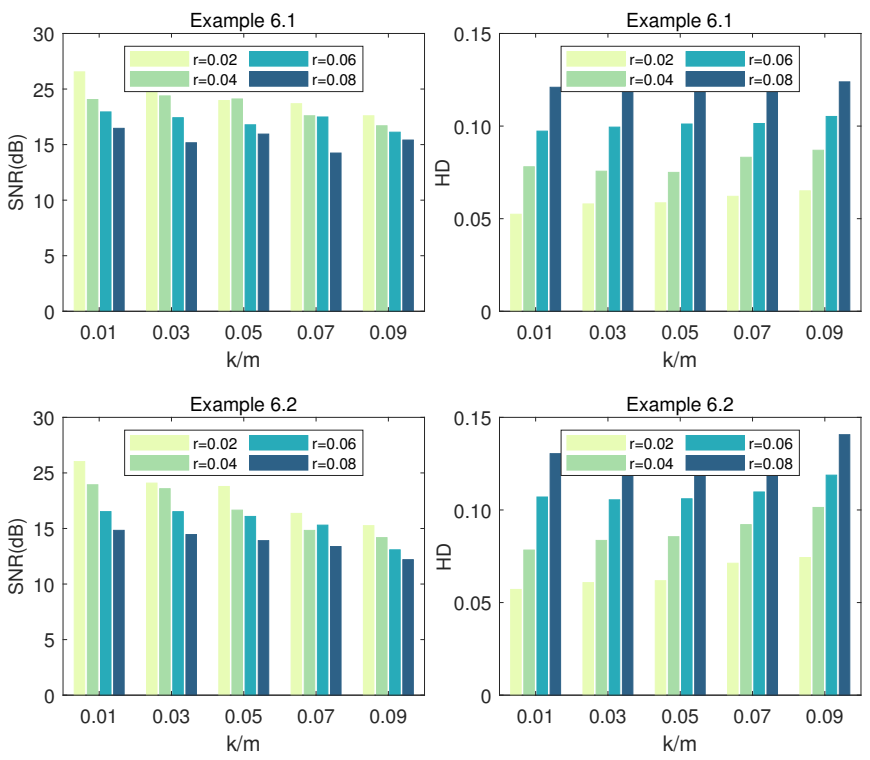

Fig. 5: Effect of $k$ for Example 6.1 and Example 6.2.

wrong recovery signs is smaller than $L$ and the number of iterations is over 50 . The other parameters for each method are chosen to be their default values. All methods are initialized by $\mathbf{x}^{0}=0$, and their final solutions are normalized to have a unit length. Finally, to make fair comparisons, we keep the first $s$ largest absolute values for PDASC and WPDASC if one obtains a solution with more than $s$ nonzero entries. Overall, all methods start with the same initial points and generate solutions with at most $s$ nonzero entries.

\section{Numerical comparisons}

We now apply the seven methods into solving two examples under different scenarios. For each scenario, we report average results over 200 instances if $n \leq 1000$ and 20 instances otherwise. Note that for each example, there are five factors $\left(n, m, s_{*}, r, v\right)$, where $v$ only makes sense for Example 6.2. In the following numerical comparisons, we shall see the effect of these factors by altering one factor while fixing the others.

(a) Effect of $s_{*}$. We first employ seven methods to solve Example 6.1 and increase $s_{*}$ from 2 to 10 with fixing $(n, m, r, v)=(500,250,0.05,0.5)$. As shown in Figure 6a, GP SP gets the highest SNR, the smallest HD and HE for each $s_{*}$, followed by AOP, PAOP and PIHT. The lines of SNR display declining trends, which means the signal is getting harder to recover when it has more nonzero components, namely, $s_{*}$ is getting bigger.

(b) Effect of $m$. To see the effect of sample size $m$, we select it from range $\{0.1,0.3, \cdots, 1.5\} n$ and fix $\left(n, s_{*}, r, v\right)=$ $(500,5,0.05,0.5)$. As shown in Figure 6b, again, GP SP outperforms the others for solving Example 6.2 since it delivers much higher SNR and lower HD and HE. It is evidently seen that all methods are behaving better along with the rising of sample size $m$ because the signal is getting easier to recover when more samples are available.

(c) Effect of $v$. We note that in Example 6.2, the larger $v$ is, the more correlated each pair of samples (i.e., rows in $\Phi$ ) are, leading to more difficult recovery. To see this, we alter $v$ from
$\{0.1,0.2, \cdots, 0.9\}$ but fix $\left(n, m, s_{*}, r\right)=(500,250,5,0.05)$, and report the average results in Figure 6c. As expected, the larger $v$ is, the more difficult the recovery is. It is observed that GPSP is quite robust to $v$ between 0.1 and 0.6 since the produced results stay steadily when $v \in[0.1,0.6]$. No matter how $v$ changes, GP SP always performs the best results among those methods.

(d) Effect of $r$. To see the effect of flipping ratio $r$, we alter it from $\{0,0.05,0.1\}$ but fix $\left(n, m, s_{*}, v\right)=(500,250,5,0.5)$. The box-plots of each method for solving Example 6.2 are presented in Figure 7 . In each box, the central mark (red line) indicates the median, the bottom, and top edges of the box indicate the 25 th and 75 th percentiles, respectively. The outliers are plotted individually using the ' + ' symbols.

As expected, the larger $r$ is, the worse performance of each method, because more correct signs are flipped. This can be testified by SNR (resp. HD and HE) whose median obtained by each method is declining (resp. rising) when $r$ ascends. Once again, GP SP behaves the best because it delivers the highest median of SNR and the lowest median of HD and HE in each box. Similar results can be observed for Example 6.1 and are omitted here.

TABLE II: Effect of the bigger values of $n$.

\begin{tabular}{|c|c|c|c|c|c|c|c|c|}
\hline & $n$ & GPSP & BIHT & PIHT & $\mathrm{AOPF}$ & PAOPF & PDASC & WPDASC \\
\hline & & \multicolumn{7}{|c|}{ Example 6.1} \\
\hline SNR & 5000 & 15.51 & 4.612 & 9.584 & 7.948 & 7.948 & -0.228 & 0.786 \\
\hline \multirow{3}{*}{$(\mathrm{dB})$} & 10000 & 12.17 & 4.431 & 9.230 & 7.464 & 7.464 & -1.755 & -1.141 \\
\hline & 15000 & 12.47 & 4.720 & 8.976 & 7.256 & 7.256 & -2.023 & -1.477 \\
\hline & 20000 & 12.89 & 4.693 & 9.095 & 7.348 & 7.348 & -2.135 & -2.111 \\
\hline \multirow[t]{4}{*}{$\mathrm{HD}$} & 5000 & 0.092 & 0.201 & 0.122 & 0.138 & 0.138 & 0.353 & 0.332 \\
\hline & 10000 & 0.106 & 0.207 & 0.125 & 0.141 & 0.141 & 0.425 & 0.394 \\
\hline & 15000 & 0.103 & 0.202 & 0.127 & 0.142 & 0.142 & 0.438 & 0.412 \\
\hline & 20000 & 0.102 & 0.201 & 0.126 & 0.143 & 0.143 & 0.444 & 0.443 \\
\hline \multirow[t]{4}{*}{$\mathrm{HE}$} & 5000 & 0.051 & 0.180 & 0.087 & 0.106 & 0.106 & 0.339 & 0.317 \\
\hline & 10000 & 0.067 & 0.185 & 0.089 & 0.109 & 0.109 & 0.417 & 0.383 \\
\hline & 15000 & 0.065 & 0.180 & 0.092 & 0.111 & 0.111 & 0.433 & 0.404 \\
\hline & 20000 & 0.062 & 0.178 & 0.091 & 0.111 & 0.111 & 0.437 & 0.437 \\
\hline TIME & 5000 & 0.147 & 0.528 & 1.187 & 0.072 & 0.014 & 0.378 & 0.473 \\
\hline \multirow[t]{4}{*}{ (s) } & 10000 & 0.580 & 4.575 & 6.124 & 0.258 & 0.056 & 1.451 & 1.698 \\
\hline & 15000 & 1.054 & 10.09 & 14.39 & 0.558 & 0.139 & 2.973 & 3.791 \\
\hline & 20000 & 1.807 & 17.78 & 24.82 & 0.978 & 0.243 & 4.788 & 6.459 \\
\hline & & \multicolumn{7}{|c|}{ Example 6.2} \\
\hline SNR & 5000 & 13.35 & 6.865 & 7.368 & 4.794 & 5.351 & -0.948 & -0.860 \\
\hline \multirow[t]{3}{*}{ (dB) } & 10000 & 11.55 & 7.699 & 7.180 & 4.980 & 5.630 & -1.857 & -1.340 \\
\hline & 15000 & 11.22 & 8.183 & 6.982 & 4.668 & 5.386 & -1.802 & -1.896 \\
\hline & 20000 & 11.67 & 8.185 & 6.942 & 4.774 & 5.549 & -2.224 & -2.324 \\
\hline \multirow[t]{4}{*}{$\mathrm{HD}$} & 5000 & 0.099 & 0.200 & 0.148 & 0.171 & 0.168 & 0.379 & 0.375 \\
\hline & 10000 & 0.106 & 0.193 & 0.148 & 0.164 & 0.163 & 0.429 & 0.405 \\
\hline & 15000 & 0.109 & 0.195 & 0.149 & 0.165 & 0.163 & 0.426 & 0.431 \\
\hline & 20000 & 0.106 & 0.191 & 0.150 & 0.162 & 0.162 & 0.446 & 0.452 \\
\hline \multirow[t]{4}{*}{$\mathrm{HE}$} & 5000 & 0.058 & 0.170 & 0.113 & 0.143 & 0.138 & 0.366 & 0.362 \\
\hline & 10000 & 0.070 & 0.164 & 0.115 & 0.137 & 0.133 & 0.422 & 0.396 \\
\hline & 15000 & 0.072 & 0.164 & 0.117 & 0.139 & 0.135 & 0.417 & 0.423 \\
\hline & 20000 & 0.069 & 0.162 & 0.117 & 0.136 & 0.133 & 0.441 & 0.448 \\
\hline TIME & 5000 & 0.155 & 0.542 & 1.274 & 2.201 & 0.086 & 0.386 & 0.474 \\
\hline \multirow[t]{3}{*}{ (s) } & 10000 & 0.566 & 4.523 & 6.448 & 13.07 & 1.600 & 1.468 & 1.736 \\
\hline & 15000 & 1.139 & 10.55 & 14.71 & 31.91 & 3.575 & 2.947 & 3.726 \\
\hline & 20000 & 1.996 & 18.39 & 25.44 & 58.05 & 10.60 & 5.103 & 7.150 \\
\hline
\end{tabular}

(e) Effect of $n$. To see the computational speed of each method, we consider some bigger values of $n$ from $\{5000,10000,15000,20000\}$ with fixing $\left(m, s_{*}, r, v\right)=$ $(n / 2, n / 100,0.05,0.5)$. The average results are recorded in Table II. Obviously, GPSP achieves the highest recovery accuracy in terms of the highest SNR, the lowest HD and 

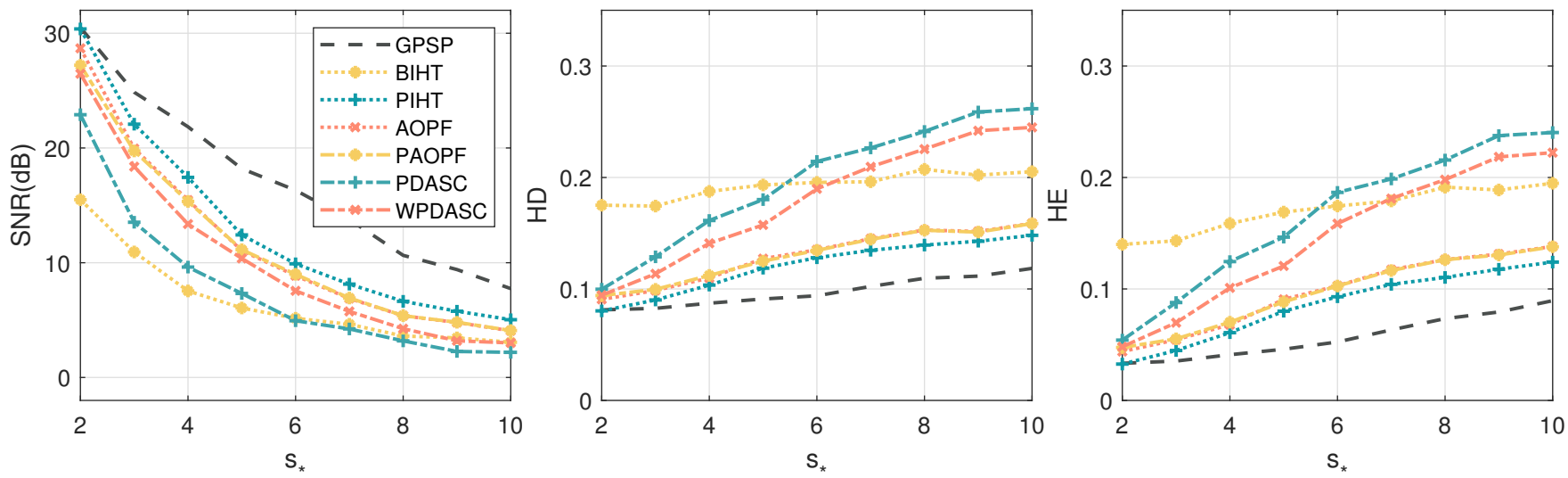

(a) Effect of $s_{*}$ for Example 6.1.
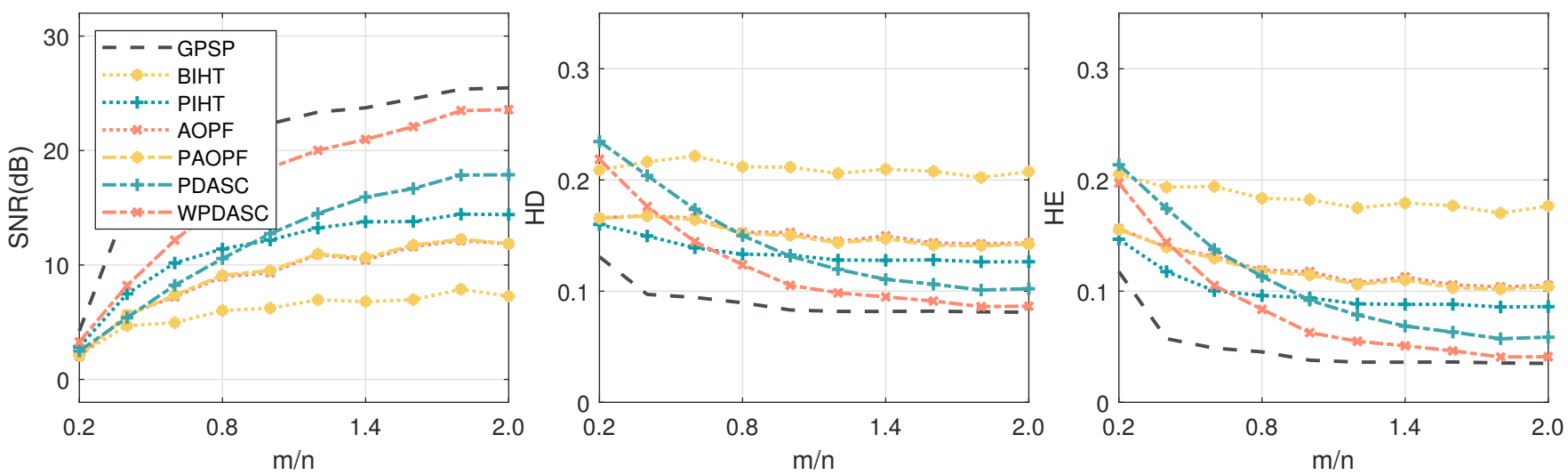

(b) Effect of $m$ for Example 6.2.
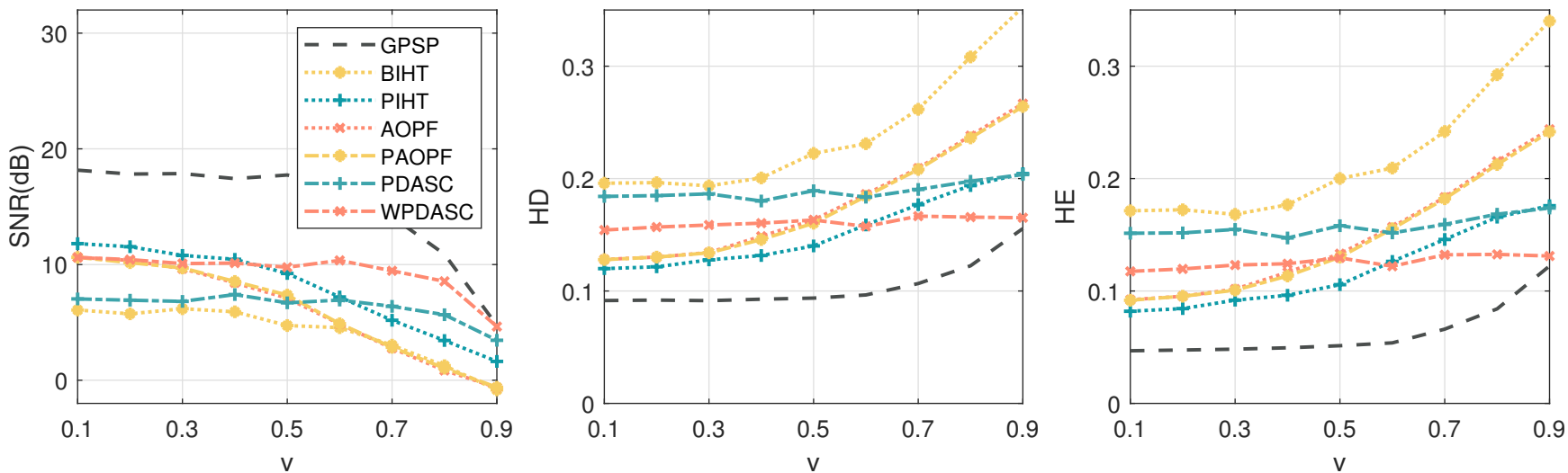

(c) Effect of $v$ for Example 6.2.

Fig. 6: Effect of $\left(s_{*}, m, v\right)$ for seven algorithms.

HE, against the other methods. For the computational speed, PAOPF runs the fastest for Example 6.1 while GPSP is the winner for Example 6.2.

In summary, we can conclude that GPSP always achieved the highest recovery accuracies and ran the fastest among all benchmark methods. There are several reasons for its high performance. Firstly, our optimization model (12) makes use of both sparsity and the number of sign flips and hence improves the recovery accuracy greatly. Moreover, as a second-order method, it is capable of enhancing the accuracy once again. Finally, it has a very low computational complexity and has been proven to terminate with finitely many steps, see (44), thereby running super fast.

\section{CONCLUSION}

In this paper, we have proposed a new approach, GPSP, that was derived from a non-convex formulation, (12), of the one-bit CS problem. The formulation has a simple objective function and exploits the double-sparsity to constrain the sparsity of the signal and the number of sign flips. Moreover, GPSP can be deemed as a second-order method. Thanks to these two aspects, GPSP is capable of delivering a high 

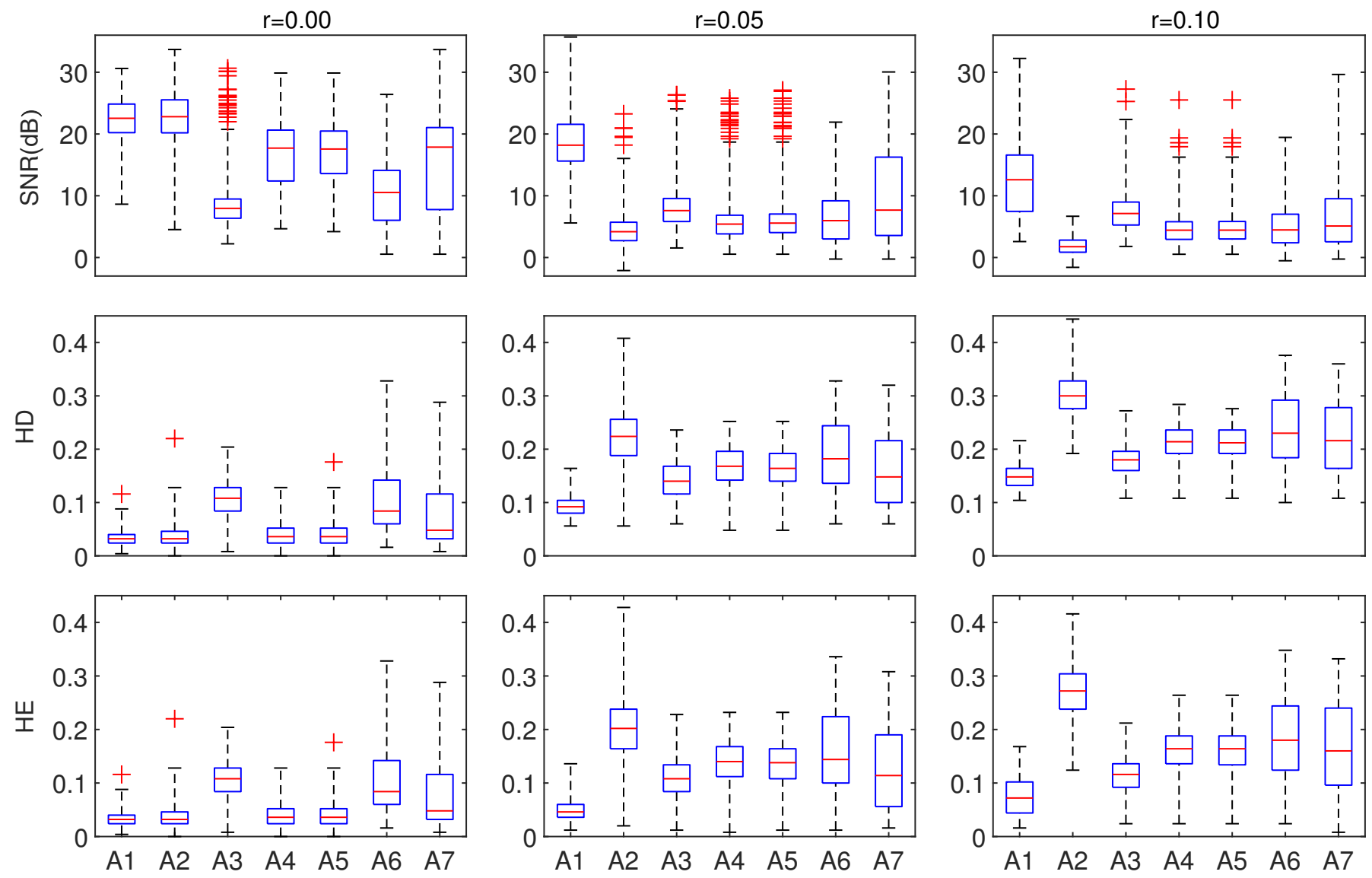

Fig. 7: Effect of $r$ for Example 6.2, where A1-A7 stand for GP SP, BIHT, PIHT, AOPF, PAOPF, PDASC, WPDASC, respectively.

reconstruction quality. To conquer the hardness resulting from the nonconvex and discrete constraints, we have established necessary and sufficient optimality conditions via the so-called $\tau$-stationarity. These optimality conditions have facilitated the design of a gradient projection subspace pursuit method GP SP, which has been shown to admit global convergence and highly efficient numerical performance both in computation time and the recovery accuracy of signals.

There are a few topics pointed out by our referees that are worth exploring further. For instance, fairly recently, authors in [47], [48], [49] succeeded in extending the noisy one-bit CS to the scenario with a time-varying threshold being given as a prior condition for the sake of further improving the recovery accuracy. Therefore, it is interesting to apply the developed techniques and the proposed algorithm into such a case.

\section{APPENDIX A}

\section{PROOFS OF ALL THEOREMS IN SECTION III}

\section{A. Proof of Lemma 3.1}

Proof: a) Necessity. Let $\mathbf{z}^{*}$ be a local minimizer of (12). Then there is a $\delta>0$ such that, for any $\mathbf{z} \in \mathcal{F} \cap N\left(\mathbf{z}^{*}, \delta\right)$,

$$
\begin{aligned}
0 & \leq \\
\stackrel{(20)}{\leq} & f(\mathbf{z})-f\left(\mathbf{z}^{*}\right) \\
& \left.\leq \nabla f\left(\mathbf{z}^{*}\right), \mathbf{z}-\mathbf{z}^{*}\right\rangle+\lambda_{\max }\left\|\mathbf{z}-\mathbf{z}^{*}\right\|^{2}=: g(\mathbf{z}) .
\end{aligned}
$$

To verify that $\mathbf{z}^{*}$ satisfies (21), we consider the four cases.
- $\left\|\mathbf{y}_{+}^{*}\right\|_{0}<k$. If there is an $i \in[m]$ such that $\left(\nabla_{\mathbf{y}} f\left(\mathbf{z}^{*}\right)\right)_{i} \neq$ 0 , then for any $t \in \mathbb{R}$, define

$$
\begin{aligned}
& \mathbf{x}_{t}:=\mathbf{x}^{*}+t \cdot 0 \\
& \mathbf{y}_{t}:=\mathbf{y}^{*}+t\left(\nabla_{\mathbf{y}} f\left(\mathbf{z}^{*}\right)\right)_{i} \cdot \mathbf{e}_{i}, \\
& \mathbf{z}_{t}:=\left(\mathbf{x}_{t} ; \mathbf{y}_{t}\right) .
\end{aligned}
$$

where $\mathbf{e}_{i} \in \mathbb{R}^{m}$ is the $i$ th column of the identity matrix. It is easy to see that $\mathbf{z}_{t} \in \mathcal{F}$ and

$$
g\left(\mathbf{z}_{t}\right)=\left(t+\lambda_{\max } t^{2}\right)\left[\left(\nabla_{\mathbf{y}} f\left(\mathbf{z}^{*}\right)\right)_{i}\right]^{2} .
$$

For any $t \in\left(\max \left\{-\delta /\left|\left(\nabla_{\mathbf{y}} f\left(\mathbf{z}^{*}\right)\right)_{i}\right|,-1 / \lambda_{\max }\right\}, 0\right)$, one can verify that $\mathbf{z}_{t} \in \mathcal{F} \cap N\left(\mathbf{z}^{*}, \delta\right)$ and $g\left(\mathbf{z}_{t}\right)<0$, contradicting with (45). Thus $\nabla_{\mathbf{y}} f\left(\mathbf{z}^{*}\right)=0$.

- $\left\|\mathbf{y}_{+}^{*}\right\|_{0}=k$. If there is an $i \in \Gamma^{*}$ such that $\left(\nabla_{\mathbf{y}} f\left(\mathbf{z}^{*}\right)\right)_{i} \neq$ 0 , then let $\mathbf{x}_{t}, \mathbf{y}_{t}$ and $\mathbf{z}_{t}$ be as (46). The same reasoning for case $\left\|\mathbf{y}_{+}^{*}\right\|_{0}<s$ enables to prove $\left(\nabla_{\mathbf{y}} f\left(\mathbf{z}^{*}\right)\right)_{i}=0$. This displays $\left(\nabla_{\mathbf{y}} f\left(\mathbf{z}^{*}\right)\right)_{\Gamma^{*}}=0$. To show $\left(\nabla_{\mathbf{y}} f\left(\mathbf{z}^{*}\right)\right)_{\bar{\Gamma}^{*}} \leq 0$, consider any given $i \in \bar{\Gamma}^{*}$. For any $t \leq 0$, let

$$
\mathbf{x}_{t}:=\mathbf{x}^{*}+t \cdot 0, \quad \mathbf{y}_{t}:=\mathbf{y}^{*}+t \cdot \mathbf{e}_{i}, \quad \mathbf{z}_{t}:=\left(\mathbf{x}_{t} ; \mathbf{y}_{t}\right) .
$$

It follows from $y_{i}^{*}=0$ and $t \leq 0$ that $\left(\mathbf{y}_{t}\right)_{+}=\mathbf{y}_{+}^{*}$ and $\left\|\mathbf{z}_{t}-\mathbf{z}^{*}\right\|=-t$. Thus, $\mathbf{z}_{t} \in \mathcal{F} \cap N\left(\mathbf{z}^{*}, \delta\right)$ for any $t \in(-\delta, 0)$. Applying (45) yields

$$
g\left(\mathbf{z}_{t}\right)=t\left(\nabla_{\mathbf{y}} f\left(\mathbf{z}^{*}\right)\right)_{i}+\lambda_{\max } t^{2} \geq 0,
$$


which implies $\left(\nabla_{\mathbf{y}} f\left(\mathbf{z}^{*}\right)\right)_{i}+\lambda_{\max } t \leq 0$. Letting $t \rightarrow 0$ derives $\left(\nabla_{\mathbf{y}} f\left(\mathbf{z}^{*}\right)\right)_{i} \leq 0$. This delivers $\left(\nabla_{\mathbf{y}} f\left(\mathbf{z}^{*}\right)\right)_{\bar{\Gamma}^{*}} \leq 0$.

- $\left\|\mathbf{x}^{*}\right\|_{0}<s$. The same reasoning for case $\left\|\mathbf{y}_{+}^{*}\right\|_{0}<k$ leads to $\nabla_{\mathbf{x}} f\left(\mathbf{z}^{*}\right)=0$.

- $\left\|\mathbf{x}^{*}\right\|_{0}=s$. The same reasoning for case $\left\|\mathbf{y}_{+}^{*}\right\|_{0}<k$ yields $\left(\nabla_{\mathbf{x}} f\left(\mathbf{z}^{*}\right)\right)_{T^{*}}=0$.

Now we conclude that case $\left\|\mathbf{y}_{+}^{*}\right\|_{0}<k$ cannot occur. If we have $\left\|\mathbf{y}_{+}^{*}\right\|_{0}<k$, then $2\left(A \mathbf{x}^{*}+\mathbf{y}^{*}-\epsilon \mathbf{1}\right)=\nabla_{\mathbf{y}} f\left(\mathbf{z}^{*}\right)=0$, resulting in $\nabla_{\mathbf{x}} f\left(\mathbf{z}^{*}\right)=2 A^{\top}\left(A \mathbf{x}^{*}+\mathbf{y}^{*}-\epsilon \mathbf{1}\right)+2 \eta \mathbf{x}^{*}=$ $2 \eta \mathbf{x}^{*}=2 \eta\left(\mathbf{x}_{T^{*}}^{*} ; 0\right)$. This together with $\left(\nabla_{\mathbf{x}} f\left(\mathbf{z}^{*}\right)\right)_{T^{*}}=0$ yields $\mathbf{x}_{T^{*}}^{*}=0$ and hence $\mathbf{x}^{*}=0$. Again by $A \mathbf{x}^{*}+\mathbf{y}^{*}-\epsilon \mathbf{1}=$ 0 , it follows $\mathbf{y}^{*}=\epsilon \mathbf{1}$ immediately. Apparently, this leads to a contradiction $k>\left\|\mathbf{y}_{+}^{*}\right\|_{0}=m \geq k$. Overall, we show (21).

b) Sufficiency. Conversely, let $\mathbf{z}^{*}$ satisfy (21). We consider the following three cases.

- $\left\|\mathbf{y}_{+}^{*}\right\|_{0}=k$. Consider a local region $N\left(\mathbf{z}^{*}, \delta_{1}\right)$ with $\delta_{1}:=$ $\min \left\{y_{i}^{*}: y_{i}^{*}>0\right\}$. Thus, for any $\mathbf{z} \in \mathcal{F} \cap N\left(\mathbf{z}^{*}, \delta_{1}\right)$, we have $y_{j}>0$ if $y_{j}^{*}>0$ and

$$
y_{i} \leq 0, \quad \forall i \in \bar{\Gamma}^{*} .
$$

In fact, if there exists an $i \in \bar{\Gamma}^{*}$ satisfying $y_{i}>0$, then $\left\|\mathbf{y}_{+}\right\|_{0} \geq\left\|\mathbf{y}_{+}^{*}\right\|_{0}+1=k+1$, which contradicts with $\mathbf{y} \in K$. Direct calculations yield

$$
\begin{array}{ll} 
& \left\langle\nabla_{\mathbf{y}} f\left(\mathbf{z}^{*}\right), \mathbf{y}-\mathbf{y}^{*}\right\rangle \\
\stackrel{(21)}{=} & \left\langle 0,\left(\mathbf{y}-\mathbf{y}^{*}\right)_{\Gamma^{*}}\right\rangle+\left\langle\left(\nabla_{\mathbf{y}} f\left(\mathbf{z}^{*}\right)\right)_{\bar{\Gamma}^{*}}, \mathbf{y}_{\bar{\Gamma}^{*}} \stackrel{(21)(48)}{\geq} 0 .\right.
\end{array}
$$

- $\left\|\mathbf{x}^{*}\right\|_{0}<s$. This yields $\left\langle\nabla_{\mathbf{x}} f\left(\mathbf{z}^{*}\right), \mathbf{x}-\mathbf{x}^{*}\right\rangle=0$ due to $\nabla_{\mathbf{x}} f\left(\mathbf{z}^{*}\right)=0$ by $(21)$.

- $\left\|\mathbf{x}^{*}\right\|_{0}=s$. Consider a local region $N\left(\mathbf{z}^{*}, \delta_{2}\right)$ with $\delta_{2}:=$ $\min \left\{\left|x_{i}^{*}\right|: x_{i}^{*} \neq 0\right\}$. For any $\mathbf{z}:=(\mathbf{x} ; \mathbf{y}) \in \mathcal{F} \cap N\left(\mathbf{z}^{*}, \delta_{2}\right)$, $x_{j} \neq 0$ if $x_{j}^{*} \neq 0$, which indicates $T^{*} \subseteq \operatorname{supp}(\mathbf{x})$. This together with $\mathbf{x} \in S$ that $\|\mathbf{x}\|_{0} \leq s=\left|T^{*}\right|$ suffices to

$$
\operatorname{supp}(\mathbf{x})=T^{*} .
$$

Now, one can verify that

$$
\begin{array}{ll} 
& \left\langle\nabla_{\mathbf{x}} f\left(\mathbf{z}^{*}\right), \mathbf{x}-\mathbf{x}^{*}\right\rangle \\
\stackrel{(21,49)}{=} & \left\langle 0,\left(\mathbf{x}-\mathbf{x}^{*}\right)_{T^{*}}\right\rangle+\left\langle\left(\nabla_{\mathbf{x}} f\left(\mathbf{z}^{*}\right)\right)_{\bar{T}^{*}}, 0\right\rangle=0 .
\end{array}
$$

Using the above facts, for any $\mathbf{z} \in \mathcal{F} \cap N\left(\mathbf{z}^{*}, \min \left\{\delta_{1}, \delta_{2}\right\}\right)$, it follows from (20) that

$$
\begin{aligned}
& f(\mathbf{z})-f\left(\mathbf{z}^{*}\right)-\left\|\mathbf{z}-\mathbf{z}^{*}\right\|_{H}^{2} \\
= & \left\langle\nabla_{\mathbf{x}} f\left(\mathbf{z}^{*}\right), \mathbf{x}-\mathbf{x}^{*}\right\rangle+\left\langle\nabla_{\mathbf{y}} f\left(\mathbf{z}^{*}\right), \mathbf{y}-\mathbf{y}^{*}\right\rangle \geq 0 .
\end{aligned}
$$

Therefore, $\mathbf{z}^{*}$ is the unique global minimizer of $\min \{f(\mathbf{z})$ : $\left.\mathbf{z} \in \mathcal{F} \cap N\left(\mathbf{z}^{*}, \min \left\{\delta_{1}, \delta_{2}\right\}\right)\right\}$, which means it is the unique local minimizer of problem (12).

\section{B. Proof of Lemma 3.2} alent to

Proof: A $\tau$-stationary point satisfies (23) which is equiv-

$$
\begin{cases}\mathbf{x}^{*} \in & \Pi_{S}\left(\mathbf{x}^{*}-\tau \nabla_{\mathbf{x}} f\left(\mathbf{z}^{*}\right)\right), \\ \mathbf{y}^{*} \in & \Pi_{K}\left(\mathbf{y}^{*}-\tau \nabla_{\mathbf{y}} f\left(\mathbf{z}^{*}\right)\right) .\end{cases}
$$

Therefore, we show the equivalence between (24) and (50). For $\mathrm{x}^{*}$ part, this can be guaranteed by [50, Lemma 2.2]. For $\mathbf{y}^{*}$ part, projection (18) enables to show that $(24) \Rightarrow(50)$. So we only prove $(50) \Rightarrow(24)$. Let $\lambda^{*}:=\nabla_{\mathbf{y}} f\left(\mathbf{z}^{*}\right)$. It follows from (18) that

$$
\begin{aligned}
\mathbf{y}^{*} & \in \Pi_{K}\left(\mathbf{y}^{*}-\tau \lambda^{*}\right) \\
& =\left\{\left[\begin{array}{c}
\mathbf{y}_{\Gamma}^{*}-\tau \lambda_{\Gamma}^{*} \\
0
\end{array}\right]: \Gamma \in \Theta\left(\mathbf{y}^{*}-\tau \lambda^{*} ; k\right)\right\} .
\end{aligned}
$$

This derives $\left\|\mathbf{y}_{+}^{*}\right\|_{0} \leq k$, and for any $\Gamma \in \Theta\left(\mathbf{y}^{*}-\tau \lambda^{*} ; k\right)$,

$$
\mathbf{y}_{\bar{\Gamma}}^{*}=0, \quad \lambda_{\Gamma}^{*}=0, \quad \mathbf{y}^{*}-\tau \lambda^{*}=\left[\mathbf{y}_{\Gamma}^{*} ;-\tau \lambda_{\bar{\Gamma}}^{*}\right],
$$

which together with the definition of $\Theta\left(\mathbf{y}^{*}-\tau \lambda^{*} ; k\right)$ in (17) gives rise to

$$
\Gamma=\Gamma_{k} \cup \Gamma_{-}=\operatorname{supp}\left(\mathbf{y}^{*}\right), \quad \bar{\Gamma}=\left(\Gamma_{+} \backslash \Gamma_{k}\right) \cup \Gamma_{0},
$$

where $\Gamma_{+}, \Gamma_{-}$and $\Gamma_{0}$ are defined as (16) in which $\mathbf{y}$ is replaced by $\mathbf{y}^{*}-\tau \lambda^{*}$. On index set $\bar{\Gamma}$, all elements $y_{i}^{*}-\tau \lambda_{i}^{*}=$ $-\tau \lambda_{i}^{*} \geq 0$, namely, $\lambda_{i}^{*} \leq 0, i \in \bar{\Gamma}$.

Now we claim that case $\left\|\mathbf{y}_{+}^{*}\right\|_{0}<k$ cannot happen. In fact, if $\left\|\mathbf{y}_{+}^{*}\right\|_{0}<k$ and suppose there is an $i \in \bar{\Gamma}$ such that $\lambda_{i}^{*}<0$, then $\mathbf{y}^{*}-\tau \lambda^{*}$ has at least $\left\|\mathbf{y}_{+}^{*}\right\|_{0}+1 \leq k$ positive entries and thus

$$
\left\|\mathbf{y}_{+}^{*}\right\|_{0}=\left\|\left(\Pi_{K}\left(\mathbf{y}^{*}-\tau \lambda^{*}\right)\right)_{+}\right\|_{0} \geq\left\|\mathbf{y}_{+}^{*}\right\|_{0}+1 .
$$

This is a contradiction. So, $\lambda_{\bar{\Gamma}}^{*}=0$, leading to $\lambda^{*}=$ $\nabla_{\mathbf{y}} f\left(\mathbf{z}^{*}\right)=0$ by (51). Then, same reasoning to show the necessity of Lemma 3.1 allows us to claim that case $\left\|\mathbf{y}_{+}^{*}\right\|_{0}<k$ cannot happen. Therefore, we have $\left\|\mathbf{y}_{+}^{*}\right\|_{0}=k$. Then (24) is satisfied for any $j \in \operatorname{supp}\left(\mathbf{y}^{*}\right)=\Gamma$ due to $\lambda_{\Gamma}^{*}=0$. For $j \notin \operatorname{supp}\left(\mathbf{y}^{*}\right)$, namely, $j \in \bar{\Gamma}$, the definition of $\Gamma_{k}$ in (17) yields

$$
0 \leq y_{j}^{*}-\tau \lambda_{j}^{*} \leq y_{i}^{*}-\tau \lambda_{i}^{*}, \quad \forall i \in \Gamma_{k},
$$

which together with $\Gamma_{k} \subseteq \Gamma$ and (51) results in

$$
0 \leq-\tau \lambda_{j}^{*} \leq y_{i}^{*}, \quad \forall i \in \Gamma_{k} .
$$

Hence, $-\left\|\mathbf{y}_{+}^{*}\right\|_{[k]}=-\min _{i \in \Gamma_{k}} y_{i}^{*} \leq \tau \lambda_{j}^{*} \leq 0, \forall j \in \bar{\Gamma}(j \notin$ $\left.\operatorname{supp}\left(\mathbf{y}^{*}\right)\right)$, showing (24).

\section{Proof of Theorem 3.1}

Proof: Let $\mathbf{z}^{*}$ be a global minimizer. If it is not a $\tau$ stationary point with $0<\tau \leq 1 /\left(2 \lambda_{\max }\right)$, then we have condition $\overline{\mathbf{z}}\left(\neq \mathbf{z}^{*}\right) \in \Pi_{\mathcal{F}}\left(\mathbf{z}^{*}-\tau \nabla f\left(\mathbf{z}^{*}\right)\right)$. Thus,

$$
\left\|\overline{\mathbf{z}}-\left(\mathbf{z}^{*}-\tau \nabla f\left(\mathbf{z}^{*}\right)\right)\right\|^{2}<\left\|\mathbf{z}^{*}-\left(\mathbf{z}^{*}-\tau \nabla f\left(\mathbf{z}^{*}\right)\right)\right\|^{2},
$$

which suffices to

$$
2 \tau\left\langle\nabla f\left(\mathbf{z}^{*}\right), \overline{\mathbf{z}}-\mathbf{z}^{*}\right\rangle<-\left\|\overline{\mathbf{z}}-\mathbf{z}^{*}\right\|^{2} .
$$

Together with (20) and $0<\tau \leq 1 /\left(2 \lambda_{\max }\right)$ derives

$$
\begin{aligned}
f(\overline{\mathbf{z}})-f\left(\mathbf{z}^{*}\right) & \leq\left\langle\nabla f\left(\mathbf{z}^{*}\right), \overline{\mathbf{z}}-\mathbf{z}^{*}\right\rangle+\lambda_{\max }\left\|\overline{\mathbf{z}}-\mathbf{z}^{*}\right\|^{2} \\
& <\left(\lambda_{\max }-1 /(2 \tau)\right)\left\|\overline{\mathbf{z}}-\mathbf{z}^{*}\right\|^{2} \leq 0 .
\end{aligned}
$$

It contradicts the global optimality of $\mathbf{z}^{*}$. Therefore, $\mathbf{z}^{*}$ is a $\tau$-stationary point with $0<\tau \leq 1 /\left(2 \lambda_{\max }\right)$.

Conversely, let $\mathbf{z}^{*}$ be a $\tau$-stationary point with $\tau \geq$ $1 /\left(2 \lambda_{\min }\right)$. The definition of $\Pi_{\mathcal{F}}$ and (23) imply

$$
\left\|\mathbf{z}^{*}-\left(\mathbf{z}^{*}-\tau \nabla f\left(\mathbf{z}^{*}\right)\right)\right\|^{2} \leq\left\|\mathbf{z}-\left(\mathbf{z}^{*}-\tau \nabla f\left(\mathbf{z}^{*}\right)\right)\right\|^{2},
$$


for any $\mathbf{z} \in \mathcal{F}$, delivering $2 \tau\left\langle\nabla f\left(\mathbf{z}^{*}\right), \mathbf{z}-\mathbf{z}^{*}\right\rangle \geq-\left\|\mathbf{z}-\mathbf{z}^{*}\right\|^{2}$. This and (20) yield

$$
\begin{aligned}
f(\mathbf{z})-f\left(\mathbf{z}^{*}\right) & \geq\left\langle\nabla f\left(\mathbf{z}^{*}\right), \mathbf{z}-\mathbf{z}^{*}\right\rangle+\lambda_{\min }\left\|\mathbf{z}-\mathbf{z}^{*}\right\|^{2} \\
& \geq\left(\lambda_{\min }-1 /(2 \tau)\right)\left\|\mathbf{z}-\mathbf{z}^{*}\right\|^{2} .
\end{aligned}
$$

Since $\tau \geq 1 /\left(2 \lambda_{\min }\right)$, the above relation shows the global optimality of $\mathbf{z}^{*}$ to (12).

\section{Proof of Corollary 3.1}

Proof: Since $\mathbf{z}^{*}$ is a local minimizer of problem (12), it satisfies (21). Then it follows

$$
\begin{aligned}
& \left(\nabla_{\mathbf{y}} f\left(\mathbf{z}^{*}\right)\right)_{\bar{\Gamma}^{*}}=2\left(A_{\bar{\Gamma}^{*} T^{*}} \mathbf{x}_{T^{*}}^{*}-\epsilon \mathbf{1}\right) \leq 0, \\
& \left(\nabla_{\mathbf{y}} f\left(\mathbf{z}^{*}\right)\right)_{\Gamma^{*}}=2\left(A_{\Gamma^{*} T^{*}} \mathbf{x}_{T^{*}}^{*}+\mathbf{y}_{\Gamma^{*}}^{*}-\epsilon \mathbf{1}\right)=0,
\end{aligned}
$$

and thus

$$
\begin{aligned}
& \left(\nabla_{\mathbf{x}} f\left(\mathbf{z}^{*}\right)\right)_{\bar{T}^{*}} \stackrel{\stackrel{(53)}{=}}{=} 2 A_{\bar{\Gamma}^{*} \bar{T}^{*}}^{\top}\left(A_{\bar{\Gamma}^{*} T^{*}} \mathbf{x}_{T^{*}}^{*}-\epsilon \mathbf{1}\right), \\
& \left(\nabla_{\mathbf{x}} f\left(\mathbf{z}^{*}\right)\right)_{T^{*}} \stackrel{(53)}{=} 2 A_{\bar{\Gamma}^{*} T^{*}}^{\top}\left(A_{\bar{\Gamma}^{*} T^{*}} \mathbf{x}_{T^{*}}^{*}-\epsilon \mathbf{1}\right)+2 \eta \mathbf{x}_{T^{*}}^{*} \\
& \stackrel{(21)}{=} 0 \text {. }
\end{aligned}
$$

Using last equation (55) results in

$$
\left(A_{\bar{\Gamma}^{*} T^{*}} A_{\bar{\Gamma}^{*} T^{*}}^{\top}+\eta I\right)\left(A_{\bar{\Gamma}^{*} T^{*}} \mathbf{x}_{T^{*}}^{*}-\epsilon \mathbf{1}\right)=-\eta \epsilon \mathbf{1} .
$$

This derives that

$$
\begin{aligned}
\left(\nabla_{\mathbf{y}} f\left(\mathbf{z}^{*}\right)\right)_{\bar{\Gamma}^{*}} & \stackrel{(52)}{=} 2\left(A_{\bar{\Gamma}^{*} T^{*}} \mathbf{x}_{T^{*}}^{*}-\epsilon \mathbf{1}\right) \\
= & -2 \eta \epsilon\left(A_{\bar{\Gamma}^{*} T^{*}} A_{\bar{\Gamma}^{*} T^{*}}^{\top}+\eta I\right)^{-1} \mathbf{1} \\
= & -\boldsymbol{\xi}^{*} .
\end{aligned}
$$

Then it is easy to see that $\boldsymbol{\xi}^{*} \geq 0$ by (52) and

$$
\forall i \in \bar{\Gamma}^{*}: \quad-\tau_{*}\left(\nabla_{\mathbf{y}} f\left(\mathbf{z}^{*}\right)\right)_{i} \stackrel{(56)}{=} \tau_{*} \xi_{i}^{*} \stackrel{(25)}{\leq}\left\|\mathbf{y}_{+}^{*}\right\|_{[k]} .
$$

If $\left\|\mathbf{x}^{*}\right\|_{0}=s$ then $\left\|\mathbf{x}^{*}\right\|_{[s]}>0$ and thus for $\forall i \in \bar{T}^{*}$

$$
\tau_{*}\left|\left(\nabla_{\mathbf{x}} f\left(\mathbf{z}^{*}\right)\right)_{i}\right| \stackrel{(54)}{=} \tau_{*}\left|\left(A_{\Gamma^{*} T^{*}}^{\top} \boldsymbol{\xi}^{*}\right)_{i}\right| \stackrel{(25)}{\leq}\left\|\mathbf{x}^{*}\right\|_{[s]} .
$$

If $\tau_{*} \geq 1 /\left(2 \lambda_{\min }\right)$, then $\mathbf{z}^{*}$ is also a global minimizer by Theorem 3.1. The whole proof is finished.

\section{APPENDIX B}

\section{PROOFS OF ALL THEOREMS IN SECTION IV}

\section{A. Proof of Lemma 4.2}

Proof: Conditions $\|\widehat{\mathbf{x}}\|=1$ and $\|\widehat{\mathbf{x}}\|_{0} \leq s$ are clearly true. We only need to show that $\left\|(-A \widehat{\mathbf{x}})_{+}\right\|_{0} \leq k$, which is equivalent to prove that $\left\|\left(-A \mathbf{x}^{*}\right)_{+}\right\|_{0} \leq k$. For notational simplicity, denote $B:=A_{\bar{\Gamma}^{*} T^{*}}$ and $D:=\left(B B^{\top}+\eta I\right)$. By noting that $A \mathbf{x}^{*}=\left(A_{\Gamma^{*} T^{*}} \mathbf{x}_{T^{*}}^{*} ; B \mathbf{x}_{T^{*}}^{*}\right)$, we next show that

$$
\left\|\left(-A_{\Gamma^{*} T^{*}} \mathbf{x}_{T^{*}}^{*}\right)_{+}\right\|_{0} \leq k, \quad B \mathbf{x}_{T^{*}}^{*} \geq 0,
$$

as it can claim $\left\|\left(-A \mathrm{x}^{*}\right)_{+}\right\|_{0} \leq k$ immediately.

Since $\mathbf{z}^{*}$ is a local minimizer of problem (12), it satisfies (24) with $\tau=\tau_{*}$, thereby leading to (52-55). From condition (53), i.e., $\mathbf{y}_{\Gamma^{*}}^{*}=\epsilon \mathbf{1}-A_{\Gamma^{*} T^{*}} \mathbf{x}_{T^{*}}^{*}$, it follows $\left\|\left(\epsilon \mathbf{1}-A_{\Gamma^{*} T^{*}} \mathbf{x}_{T^{*}}^{*}\right)_{+}\right\|_{0}=k$ due to $\left\|\left(\mathbf{y}_{\Gamma^{*}}^{*}\right)_{+}\right\|_{0}=\left\|\mathbf{y}_{+}^{*}\right\|_{0}=k$, thereby yielding

$$
\left\|\left(-A_{\Gamma^{*} T^{*}} \mathbf{x}_{T^{*}}^{*}\right)_{+}\right\|_{0} \leq k .
$$

Using equation (55) results in

$$
\begin{aligned}
\mathbf{x}_{T^{*}}^{*} & =\epsilon\left(B^{\top} B+\eta I\right)^{-1} B^{\top} \mathbf{1} \\
& =(\epsilon / \eta)\left(I-B^{\top} D^{-1} B\right) B^{\top} \mathbf{1} \\
& =(\epsilon / \eta) B^{\top}\left(I-D^{-1} B B^{\top}\right) \mathbf{1} \\
& =(\epsilon / \eta) B^{\top}\left(I-D^{-1}\left(B B^{\top}+\eta I-\eta I\right)\right) \mathbf{1} \\
& =\epsilon B^{\top} D^{-1} \mathbf{1},
\end{aligned}
$$

where the second equation is due to the Woodbury matrix identity, namely, $(I+U C V)^{-1}=I-U\left(C^{-1}+V U\right)^{-1} V$. Therefore, we obtain

$$
\begin{aligned}
\epsilon \mathbf{1}-B \mathbf{x}_{T^{*}}^{*} & =\epsilon \mathbf{1}-\epsilon B B^{\top} D^{-1} \mathbf{1} \\
& =\epsilon \mathbf{1}-\epsilon\left(B B^{\top}+\eta I-\eta I\right) D^{-1} \mathbf{1} \\
& =\epsilon \eta D^{-1} \mathbf{1} \\
& =\epsilon \eta U \operatorname{Diag}\left(\left(\lambda_{1}+\eta\right)^{-1} \cdots\left(\lambda_{r}+\eta\right)^{-1}\right) U^{\top} \mathbf{1},
\end{aligned}
$$

where the last equation used the eigenvalue decomposition of $B B^{\top}=U \operatorname{Diag}\left(\lambda_{1} \cdots \lambda_{r}\right) U^{\top}$ with $\lambda_{1} \geq \cdots \geq \lambda_{r}$ and $r=\left|\bar{\Gamma}^{*}\right|$. We note that $\lambda_{r} \geq c_{A} \geq 0$. If $\lambda_{r}=0$, then $\eta=0$ by (29) and thus $\epsilon \mathbf{1}-B \mathbf{x}_{T^{*}}^{*}=0$. This shows $B \mathbf{x}_{T^{*}}^{*}=\epsilon \mathbf{1}>0$. Now we focus on case $\lambda_{r}>0$. It is easy to see that $r=$ $m-\left|\Gamma^{*}\right| \leq m-\left\|\mathbf{y}_{+}^{*}\right\|_{0}=m-k$. Using these facts allows us to derive that

$$
\begin{aligned}
0 \stackrel{(52)}{\leq} \epsilon \mathbf{1}-B \mathbf{x}_{T^{*}}^{*} & \leq\left\|\epsilon \mathbf{1}-B \mathbf{x}_{T^{*}}^{*}\right\|_{\infty} \mathbf{1} \\
& \leq\left\|\epsilon \mathbf{1}-B \mathbf{x}_{T^{*}}^{*}\right\| \mathbf{1} \\
& \leq \epsilon \eta /\left(\lambda_{r}+\eta\right)\left\|U^{\top} \mathbf{1}\right\| \mathbf{1} \\
& =\epsilon \eta \sqrt{r} /\left(\lambda_{r}+\eta\right) \mathbf{1} \\
& \leq \epsilon \eta \sqrt{m-k} /\left(\lambda_{r}+\eta\right) \mathbf{1} \\
& \leq \epsilon \mathbf{1},
\end{aligned}
$$

resulting in $B \mathbf{x}_{T^{*}}^{*} \geq 0$ as well.

\section{B. Proof of Theorem 4.1}

Proof: It follows from [28, Lemma 4] that

$$
\frac{1}{m}\left\|\operatorname{sgn}\left(\Phi \mathbf{x}^{\text {true }}+\varepsilon\right)-\operatorname{sgn}\left(\Phi \mathbf{x}^{\text {true }}\right)\right\|_{0} \leq \frac{\varrho}{2}+\delta
$$

with a probability higher than $1-e^{-2 m \delta^{2}}$. Since $\Phi$ is generated as Lemma 4.1 with $m \geq m(2 s, \delta, \theta)$ for a fixed $\theta \in[0,1]$ and $\delta \in(0,1)$, mapping $\operatorname{sgn}(\cdot)$ is then a $\mathrm{B} \delta \mathrm{SE}$ of order $2 s$ with probability exceeding $1-\theta$ by Lemma 4.1. Hence,

$$
d\left(\widehat{\mathbf{x}}, \mathbf{x}^{\text {true }}\right) \leq \frac{1}{m}\left\|\operatorname{sgn}(\Phi \widehat{\mathbf{x}})-\operatorname{sgn}\left(\Phi \mathbf{x}^{\text {true }}\right)\right\|_{0}+\delta .
$$

We have shown $\left\|(-A \widehat{\mathbf{x}})_{+}\right\|_{0} \leq k$ in (30), which together with $A=\operatorname{Diag}(\mathbf{c}) \Phi$ implies $\|\operatorname{sgn}(\Phi \widehat{\mathbf{x}})-\mathbf{c}\|_{0} \leq k$. Moreover, it is easy to see that $\left\|\mathbf{c}-\operatorname{sgn}\left(\Phi \mathbf{x}^{\text {true }}+\varepsilon\right)\right\|_{0} \leq k$. Overall, using these facts allows us to derive that

$$
\begin{aligned}
& d\left(\widehat{\mathbf{x}}, \mathbf{x}^{\text {true }}\right) \\
\stackrel{(58)}{\leq} & \frac{1}{m}\left\|\operatorname{sgn}(\Phi \widehat{\mathbf{x}})-\operatorname{sgn}\left(\Phi \mathbf{x}^{\text {true }}\right)\right\|_{0}+\delta \\
\leq & \frac{1}{m}\left\|\operatorname{sgn}(\Phi \widehat{\mathbf{x}})-\operatorname{sgn}\left(\Phi \mathbf{x}^{\text {true }}+\boldsymbol{\varepsilon}\right)\right\|_{0} \\
+ & \frac{1}{m}\left\|\operatorname{sgn}\left(\Phi \mathbf{x}^{\text {true }}+\varepsilon\right)-\operatorname{sgn}\left(\Phi \mathbf{x}^{\text {true }}\right)\right\|_{0}+\delta
\end{aligned}
$$




$$
\begin{aligned}
& \leq \frac{1}{m}\|\operatorname{sgn}(\Phi \widehat{\mathbf{x}})-\mathbf{c}\|_{0}+\frac{1}{m}\left\|\mathbf{c}-\operatorname{sgn}\left(\Phi \mathbf{x}^{\text {true }}+\varepsilon\right)\right\|_{0} \\
& +\frac{1}{m}\left\|\operatorname{sgn}\left(\Phi \mathbf{x}^{\text {true }}+\varepsilon\right)-\operatorname{sgn}\left(\Phi \mathbf{x}^{\text {true }}\right)\right\|_{0}+\delta \\
& \stackrel{(57)}{\leq} \frac{k}{m}+\frac{k}{m}+\frac{\varrho}{2}+2 \delta=2 \phi
\end{aligned}
$$

Finally, it is easy to see that, for any $\|\mathbf{u}\|=\|\mathbf{v}\|=1$,

$$
d(\mathbf{u}, \mathbf{v})=\frac{1}{\pi} \arccos \langle\mathbf{u}, \mathbf{v}\rangle=\frac{1}{\pi} \arccos \frac{2-\|\mathbf{u}-\mathbf{v}\|^{2}}{2}
$$

due to $0 \leq \phi \leq 1 / 2$, which suffices to

$$
\begin{aligned}
d(\mathbf{u}, \mathbf{v}) \leq 2 \phi \Longrightarrow\|\mathbf{u}-\mathbf{v}\|^{2} & \leq 2-2 \cos (2 \phi \pi) \\
& =2-2\left(1-2 \sin ^{2}(\phi \pi)\right) \\
& =4 \sin ^{2}(\phi \pi) .
\end{aligned}
$$

This together with (59) displays the desired result.

\section{APPENDIX C}

\section{Proofs of ALL THEOREMS IN SECTION V}

\section{A. Proof of Lemma 5.1}

Proof: It follows from $\mathbf{z}^{\ell}(\tau) \in \Pi_{\mathcal{F}}\left(\mathbf{z}^{\ell}-\tau \nabla f\left(\mathbf{z}^{\ell}\right)\right)$ that

$$
\left\|\mathbf{z}^{\ell}(\tau)-\left(\mathbf{z}^{\ell}-\tau \nabla f\left(\mathbf{z}^{\ell}\right)\right)\right\|^{2} \leq\left\|\mathbf{z}^{\ell}-\left(\mathbf{z}^{\ell}-\tau \nabla f\left(\mathbf{z}^{\ell}\right)\right)\right\|^{2},
$$

which results in

$$
2 \tau\left\langle\nabla f\left(\mathbf{z}^{\ell}\right), \mathbf{z}^{\ell}(\tau)-\mathbf{z}^{\ell}\right\rangle \leq-\left\|\mathbf{z}^{\ell}(\tau)-\mathbf{z}^{\ell}\right\|^{2} .
$$

Combining with (20) leads to

$$
\begin{aligned}
& f\left(\mathbf{z}^{\ell}(\tau)\right) \\
\leq & f\left(\mathbf{z}^{\ell}\right)+\left\langle\nabla f\left(\mathbf{z}^{\ell}\right), \mathbf{z}^{\ell}(\tau)-\mathbf{z}^{\ell}\right\rangle+\lambda_{\max }\left\|\mathbf{z}^{\ell}(\tau)-\mathbf{z}^{\ell}\right\|^{2} \\
\leq & f\left(\mathbf{z}^{\ell}\right)-\left(1 /(2 \tau)-\lambda_{\max }\right)\left\|\mathbf{z}^{\ell}(\tau)-\mathbf{z}^{\ell}\right\|^{2} \\
\leq & f\left(\mathbf{z}^{\ell}\right)-\rho\left\|\mathbf{z}^{\ell}(\tau)-\mathbf{z}^{\ell}\right\|^{2},
\end{aligned}
$$

where the last inequality is from $0<\tau \leq 1 /\left(2 \rho+2 \lambda_{\max }\right)$. Invoking the Armijo-type step size rule, one has $\tau_{\ell} \geq \beta /(2 \rho+$ $\left.2 \lambda_{\max }\right)$, which by $\tau_{\ell} \leq 1$ proves the desired assertion.

\section{B. Proof of Lemma 5.2}

Proof: i) By Lemma 5.1 and $\mathbf{u}^{\ell}=\mathbf{z}^{\ell}\left(\tau_{\ell}\right)$, we have

$$
f\left(\mathbf{u}^{\ell}\right) \leq f\left(\mathbf{z}^{\ell}\right)-\rho\left\|\mathbf{u}^{\ell}-\mathbf{z}^{\ell}\right\|^{2} .
$$

By the framework of Algorithm 1, if $\mathbf{z}^{\ell+1}=\mathbf{u}^{\ell}$, then the above condition implies,

$$
\begin{aligned}
f\left(\mathbf{z}^{\ell+1}\right) & \leq f\left(\mathbf{z}^{\ell}\right)-\rho\left\|\mathbf{u}^{\ell}-\mathbf{z}^{\ell}\right\|^{2} \\
& =f\left(\mathbf{z}^{\ell}\right)-\rho\left\|\mathbf{z}^{\ell+1}-\mathbf{z}^{\ell}\right\|^{2} .
\end{aligned}
$$

If $\mathbf{z}^{\ell+1}=\mathbf{v}^{\ell}$, then we obtain

$$
\begin{aligned}
f\left(\mathbf{z}^{\ell+1}\right) & =f\left(\mathbf{v}^{\ell}\right) \\
& \leq f\left(\mathbf{u}^{\ell}\right)-\rho\left\|\mathbf{z}^{\ell+1}-\mathbf{u}^{\ell}\right\|^{2} \\
& \leq f\left(\mathbf{z}^{\ell}\right)-\rho\left\|\mathbf{u}^{\ell}-\mathbf{z}^{\ell}\right\|^{2}-\rho\left\|\mathbf{z}^{\ell+1}-\mathbf{u}^{\ell}\right\|^{2} \\
& \leq f\left(\mathbf{z}^{\ell}\right)-(\rho / 2)\left\|\mathbf{z}^{\ell+1}-\mathbf{z}^{\ell}\right\|^{2}
\end{aligned}
$$

where the second and last inequalities used (61) and a fact $\|\mathbf{a}+\mathbf{b}\|^{2} \leq 2\|\mathbf{a}\|^{2}+2\|\mathbf{b}\|^{2}$ for all vectors $\mathbf{a}$ and $\mathbf{b}$. Both cases lead to

$$
\begin{aligned}
& f\left(\mathbf{z}^{\ell+1}\right) \leq f\left(\mathbf{z}^{\ell}\right)-(\rho / 2)\left\|\mathbf{z}^{\ell+1}-\mathbf{z}^{\ell}\right\|^{2}, \\
& f\left(\mathbf{z}^{\ell+1}\right) \leq f\left(\mathbf{z}^{\ell}\right)-\rho\left\|\mathbf{u}^{\ell}-\mathbf{z}^{\ell}\right\|^{2} .
\end{aligned}
$$

Therefore, $\left\{f\left(\mathbf{z}^{\ell}\right)\right\}$ is a non-increasing sequence, and thus

$$
\max \left\{\left\|A \mathbf{x}^{\ell}-\epsilon \mathbf{1}+\mathbf{y}^{\ell}\right\|^{2}, \eta\left\|\mathbf{x}^{\ell}\right\|^{2}\right\} \leq f\left(\mathbf{z}^{\ell}\right) \leq f\left(\mathbf{z}^{0}\right),
$$

which indicates the boundedness of $\left\{\mathbf{x}^{\ell}\right\}$ and $\left\{\mathbf{y}^{\ell}\right\}$, and so that of $\left\{\mathbf{z}^{\ell}\right\}$. The non-increasing property in (64) and $f \geq 0$ also give rise to

$$
\begin{aligned}
& \sum_{\ell \geq 0} \max \left\{(\rho / 2)\left\|\mathbf{z}^{\ell+1}-\mathbf{z}^{\ell}\right\|^{2}, \rho\left\|\mathbf{u}^{\ell}-\mathbf{z}^{\ell}\right\|^{2}\right\} \\
\leq & \sum_{\ell \geq 0}\left[f\left(\mathbf{z}^{\ell}\right)-f\left(\mathbf{z}^{\ell+1}\right)\right] \\
= & f\left(\mathbf{z}^{0}\right)-\lim _{\ell \rightarrow \infty} f\left(\mathbf{z}^{\ell+1}\right) \leq f\left(\mathbf{z}^{0}\right) .
\end{aligned}
$$

The above condition suffices to $\lim _{\ell \rightarrow \infty}\left\|\mathbf{z}^{\ell+1}-\mathbf{z}^{\ell}\right\|=$ $\lim _{\ell \rightarrow \infty}\left\|\mathbf{u}^{\ell}-\mathbf{z}^{\ell}\right\|=0$.

ii) Let $\mathbf{z}^{*}$ be any accumulating point of $\left\{\mathbf{z}^{\ell}\right\}$. Then there exists a subset $J$ of $\{0,1,2, \cdots\}$ such that $\lim _{\ell(\in J) \rightarrow \infty} \mathbf{z}^{\ell}=$ $\mathbf{z}^{*}$. This further implies $\lim _{\ell(\in J) \rightarrow \infty} \mathbf{u}^{\ell}=\mathbf{z}^{*}$ by applying a). In addition, as stated in Lemma 5.1, we have $\left\{\tau_{\ell}\right\} \subseteq[\underline{\tau}, 1]$, which indicates that one can find a subsequence $L$ of $J$ and a scalar $\tau_{*} \in[\underline{\tau}, 1]$ such that $\left\{\tau_{\ell}: \ell \in L\right\} \rightarrow \tau_{*}$. To summarize, we have

$\mathbf{z}^{\ell} \rightarrow \mathbf{z}^{*}, \quad \mathbf{u}^{\ell} \rightarrow \mathbf{z}^{*}, \quad \tau_{\ell} \rightarrow \tau_{*} \in[\underline{\tau}, 1], \quad \ell(\in L) \rightarrow \infty$.

Let $\overline{\mathbf{z}}^{\ell}:=\mathbf{z}^{\ell}-\tau_{\ell} \nabla f\left(\mathbf{z}^{\ell}\right)$. Algorithm 1 implies

$$
\mathbf{u}^{\ell} \in \Pi_{\mathcal{F}}\left(\overline{\mathbf{z}}^{\ell}\right), \quad \lim _{\ell(\in L) \rightarrow \infty} \overline{\mathbf{z}}^{\ell}=\mathbf{z}^{*}-\tau_{*} \nabla f\left(\mathbf{z}^{*}\right)=: \overline{\mathbf{z}}^{*} .
$$

The first condition means $\mathbf{u}^{\ell} \in \mathcal{F}$ for any $\ell \geq 1$. Note that $\mathcal{F}$ is closed and $\mathbf{z}^{*}$ is the accumulating point of $\left\{\mathbf{u}^{\ell}\right\}$ by (65). Therefore, $\mathbf{z}^{*} \in \mathcal{F}$, which results in

$$
\min _{\mathbf{z} \in \mathcal{F}}\left\|\mathbf{z}-\overline{\mathbf{z}}^{*}\right\| \leq\left\|\mathbf{z}^{*}-\overline{\mathbf{z}}^{*}\right\| .
$$

If the strict inequality holds in the above condition, then there is an $\varepsilon_{0}>0$ such that

$$
\begin{aligned}
\left\|\mathbf{z}^{*}-\overline{\mathbf{z}}^{*}\right\|-\varepsilon_{0} & =\min _{\mathbf{z} \in \mathcal{F}}\left\|\mathbf{z}-\overline{\mathbf{z}}^{*}\right\| \\
& \geq \min _{\mathbf{z} \in \mathcal{F}}\left(\left\|\mathbf{z}-\overline{\mathbf{z}}^{\ell}\right\|-\left\|\overline{\mathbf{z}}^{\ell}-\overline{\mathbf{z}}^{*}\right\|\right) \\
& =\left\|\mathbf{u}^{\ell}-\overline{\mathbf{z}}^{\ell}\right\|-\left\|\overline{\mathbf{z}}^{\ell}-\overline{\mathbf{z}}^{*}\right\|
\end{aligned}
$$

where the last equality is from (66). Taking the limit of both sides of the above condition along $\ell(\in L) \rightarrow \infty$ yields $\| \mathbf{z}^{*}-$ $\overline{\mathbf{z}}^{*}\left\|-\varepsilon_{0} \geq\right\| \mathbf{z}^{*}-\overline{\mathbf{z}}^{*} \|$ by (65) and (66), a contradiction with $\varepsilon_{0}>0$. Therefore, we must have the equality holds in (67), showing that

$$
\mathbf{z}^{*} \in \Pi_{\mathcal{F}}\left(\overline{\mathbf{z}}^{*}\right)=\Pi_{\mathcal{F}}\left(\mathbf{z}^{*}-\tau_{*} \nabla f\left(\mathbf{z}^{*}\right)\right) .
$$

The above relation means the conditions in (24) hold for $\tau=$ $\tau_{*}$, then these conditions must hold for any $0<\tau \leq \underline{\tau}$ due to $\underline{\tau} \leq \tau_{*}$ from (65), namely,

$$
\mathbf{z}^{*} \in \Pi_{\mathcal{F}}\left(\mathbf{z}^{*}-\tau \nabla f\left(\mathbf{z}^{*}\right)\right),
$$

displaying that $\mathbf{z}^{*}$ is a $\tau$-stationary point of (12). 


\section{Proof of Theorem 5.1}

Proof: As shown in Lemma 5.2, one can find a subsequence of $\left\{\mathbf{z}^{\ell}\right\}$ that converges to the $\tau$-stationary point $\mathbf{z}^{*}$ with $0<\tau \leq \underline{\tau}$ of (12). Recall that a $\tau$-stationary point $\mathbf{z}^{*}$ that satisfies (24) also meets (21), which by Lemma 3.1 indicates that $\mathbf{z}^{*}$ is the unique local minimizer. In other words, $\mathbf{z}^{*}$ is an isolated local minimizer of (12). Finally, it follows from $\mathbf{z}^{*}$ being isolated, [51, Lemma 4.10] and $\lim _{\ell \rightarrow \infty}\left\|\mathbf{z}^{\ell+1}-\mathbf{z}^{\ell}\right\|=0$ by Lemma 5.2 that the whole sequence converges to $\mathbf{z}^{*}$. It is also a global minimizer due to Corollary 3.1.

\section{Proof of Theorem 5.2}

Proof: It follows Theorem 5.1 and Lemma 5.2 that both $\mathbf{z}^{\ell}(\in \mathcal{F}) \rightarrow \mathbf{z}^{*}$ and $\mathbf{u}^{\ell}(\in \mathcal{F}) \rightarrow \mathbf{z}^{*}$ and $\mathbf{z}^{*}$ is a $\tau$-stationary point. Thus $\left\|\mathbf{y}_{+}^{*}\right\|_{0}=k$. This implies $\Gamma_{+}^{\ell} \equiv \widetilde{\Gamma}_{+}^{\ell} \equiv \Gamma_{+}^{*}$ for sufficiently large $\ell$. If $\left\|\mathbf{x}^{*}\right\|_{0}=s$, then $\mathbf{z}^{\ell}(\in \mathcal{F}) \rightarrow \mathbf{z}^{*}$ indicates $T^{\ell} \equiv \widetilde{T}^{\ell} \equiv T^{*}$ for sufficiently large $\ell$. If $\left\|\mathbf{x}^{*}\right\|_{0}<s$, then by (24) that $\nabla_{\mathbf{x}} f\left(\mathbf{z}^{*}\right)=0$. This together with $\mathbf{u}^{\ell}(\in$ $\mathcal{F}) \rightarrow \mathbf{z}^{*}$ results in $\left\|\nabla_{\mathbf{x}} f\left(\mathbf{u}^{\ell}\right)\right\|<\varepsilon$. Therefore, the framework of Algorithm 1 allows us to assert that $\mathbf{z}^{\ell+1}=\mathbf{v}^{\ell}$ for all sufficiently large $\ell$ due to $0<\rho \leq \lambda_{\min }$ and

$$
\begin{aligned}
f\left(\mathbf{v}^{\ell}\right) & \stackrel{(40)}{\leq} f\left(\mathbf{u}^{\ell}\right)-\lambda_{\min }\left\|\mathbf{u}^{\ell}-\mathbf{v}^{\ell}\right\|^{2} \\
& \leq f\left(\mathbf{u}^{\ell}\right)-\rho\left\|\mathbf{u}^{\ell}-\mathbf{v}^{\ell}\right\|^{2}
\end{aligned}
$$

The above assertion means (38) is always admitted for sufficiently large $\ell$. However, updating rule (38) of $\mathbf{v}^{\ell}$ indicates

$$
T^{\ell+1} \subseteq T^{\ell}, \quad \Gamma_{+}^{\ell+1} \subseteq \Gamma_{+}^{\ell}, \quad \Gamma_{0}^{\ell+1} \supseteq \Gamma_{0}^{\ell}, \quad \Gamma_{-}^{\ell+1} \subseteq \Gamma_{-}^{\ell} .
$$

Note that these sets have finite elements. Therefore, the sequences $\left\{T^{\ell}\right\},\left\{\Gamma_{+}^{\ell}\right\},\left\{\Gamma_{0}^{\ell}\right\},\left\{\Gamma_{-}^{\ell}\right\}$ converge. In other words, there is a finite $\kappa \geq 1$ such that, for any $\ell \geq \kappa$,

$$
T^{\ell+1}=T^{\ell}, \quad \Gamma_{+}^{\ell+1}=\Gamma_{+}^{\ell}, \quad \Gamma_{0}^{\ell+1}=\Gamma_{0}^{\ell}, \quad \Gamma_{-}^{\ell+1}=\Gamma_{-}^{\ell},
$$

which implies $\Omega\left(\mathbf{z}^{\ell+1}\right)=\Omega\left(\mathbf{z}^{\ell}\right)$. This and (38) yield

$$
\begin{aligned}
\mathbf{z}^{\ell+2}=\mathbf{v}^{\ell+1} & =\operatorname{argmin}\left\{f(\mathbf{z}): \mathbf{z} \in \Omega\left(\mathbf{z}^{\ell+1}\right)\right\} \\
& =\operatorname{argmin}\left\{f(\mathbf{z}): \mathbf{z} \in \Omega\left(\mathbf{z}^{\ell}\right)\right\} \\
& =\mathbf{v}^{\ell}=\mathbf{z}^{\ell+1} .
\end{aligned}
$$

Overall, for any $\ell \geq \kappa$, we have $\mathbf{z}^{\ell}=\mathbf{z}^{\kappa}$. Recall Theorem 5.1 that whole sequence $\left\{\mathbf{z}^{\ell}\right\}$ converges to $\mathbf{z}^{*}$, which suffices to $\mathbf{z}^{*}=\lim _{\ell \rightarrow \infty} \mathbf{z}^{\ell}=\mathbf{z}^{\kappa}$.

\section{E. Proof of Theorem 5.3}

Proof: Since limit $\mathbf{z}^{*}$ is a local minimizer by Theorem 5.1, we have (32) from Theorem 4.1, namely,

$$
\left\|c_{*} \mathbf{x}^{*}-\mathbf{x}^{\text {true }}\right\| \leq 2 \sin (\phi \pi) \text {. }
$$

Theorem 5.2 ii) shows that GPSP will terminate at limit $\mathbf{z}^{*}$ within finite steps. That is, there is a finite $\kappa$ such that $\mathbf{z}^{\ell}=\mathbf{z}^{*}$ for any $\ell \geq \kappa$, which derives

$$
\left\|c_{*} \mathbf{x}^{\ell}-\mathbf{x}^{\text {true }}\right\|=\left\|c_{*} \mathbf{x}^{*}-\mathbf{x}^{\text {true }}\right\| \leq 2 \sin (\phi \pi) .
$$

Hence, the whole proof is completed.

\section{ACKNOWLEDGMENT}

The authors sincerely thank the associate editor and the five referees for their constructive comments, which have significantly improved the quality of the paper.

\section{REFERENCES}

[1] E. J. Candes and T. Tao, "Decoding by linear programming," IEEE Trans. Inf. Theory, vol. 51, no. 12, pp. 4203-4215, 2005.

[2] E. J. Candès, J. Romberg, and T. Tao, "Robust uncertainty principles: Exact signal reconstruction from highly incomplete frequency information," IEEE Trans. Inf. Theory, vol. 52, no. 2, pp. 489-509, 2006.

[3] D. L. Donoho, "Compressed sensing," IEEE Trans. Inf. Theory, vol. 52, no. 4, pp. 1289-1306, 2006.

[4] P. T. Boufounos and R. G. Baraniuk, "1-bit compressive sensing," in 42nd Аnnu. Conf. Inf. Sci. Syst. IEEE, 2008, pp. 16-21.

[5] J. Haboba, M. Mangia, R. Rovatti, and G. Setti, "An architecture for 1-bit localized compressive sensing with applications to EEG," in IEEE Biomed. Circuits Syst. Conf. IEEE, 2011, pp. 137-140.

[6] A. Movahed and M. C. Reed, "Iterative detection for compressive sensing: Turbo CS," in IEEE Int. Conf. Commun. IEEE, 2014, pp. 4518-4523.

[7] W. Tang, W. Xu, X. Zhang, and J. Lin, "A low-cost channel feedback scheme in mmwave massive mimo system," in 3rd IEEE Int. Conf. Intell. Comput. Commun. IEEE, 2017, pp. 89-93.

[8] Z. Zhou, X. Chen, D. Guo, and M. L. Honig, "Sparse channel estimation for massive MIMO with 1-bit feedback per dimension," in IEEE Wirel. Commun. Netw. Conf. IEEE, 2017, pp. 1-6.

[9] J. Meng, H. Li, and Z. Han, "Sparse event detection in wireless sensor networks using compressive sensing," in 43rd Annu. Conf. Inf. Sci. Syst. IEEE, 2009, pp. 181-185.

[10] C. Feng, S. Valaee, and Z. Tan, "Multiple target localization using compressive sensing," in IEEE Glob. Commun. Conf. IEEE, 2009, pp. $1-6$.

[11] J. Xiong and Q. Tang, "1-bit compressive data gathering for wireless sensor networks," J. Sens., vol. 2014, 2014.

[12] C.-H. Chen and J.-Y. Wu, "Amplitude-aided 1-bit compressive sensing over noisy wireless sensor networks," IEEE Wirel. Commun. Lett., vol. 4, no. 5, pp. 473-476, 2015.

[13] D. Lee, T. Sasaki, T. Yamada, K. Akabane, Y. Yamaguchi, and K. Uehara, "Spectrum sensing for networked system using 1-bit compressed sensing with partial random circulant measurement matrices," in IEEE 75th Veh. Technol. Conf. IEEE, 2012, pp. 1-5.

[14] N. Fu, L. Yang, and J. Zhang, "Sub-nyquist 1 bit sampling system for sparse multiband signals," in 22nd Eur. Signal Process. Conf. IEEE, 2014, pp. 736-740.

[15] A. Bourquard and M. Unser, "Binary compressed imaging," IEEE Trans. Image Process., vol. 22, no. 3, pp. 1042-1055, 2012.

[16] X. Dong and Y. Zhang, "A MAP approach for 1-bit compressive sensing in synthetic aperture radar imaging," IEEE Geosci. Remote Sens. Lett., vol. 12, no. 6, pp. 1237-1241, 2015.

[17] D. Marcos, T. Lasser, A. López, and A. Bourquard, "Compressed imaging by sparse random convolution," Opt. Express, vol. 24, no. 2, pp. 1269-1290, 2016.

[18] Z. Li, W. Xu, X. Zhang, and J. Lin, "A survey on one-bit compressed sensing: Theory and applications," Front. Comput. Sci., vol. 12, no. 2, pp. 217-230, 2018.

[19] Z. Qin, J. Fan, Y. Liu, Y. Gao, and G. Y. Li, "Sparse representation for wireless communications: A compressive sensing approach," IEEE Signal Process. Mag., vol. 35, no. 3, pp. 40-58, 2018.

[20] S. Gopi, P. Netrapalli, P. Jain, and A. Nori, "One-bit compressed sensing: Provable support and vector recovery," in Int. Conf. Mach. Learn. PMLR, 2013, pp. 154-162.

[21] H. Wang, X. Huang, Y. Liu, H. Sabine Van, and W. Qun, "Binary reweighted 11-norm minimization for one-bit compressed sensing," in 8th Int. Jt. Conf. Biomed. Eng. Syst. Technol., 2015.

[22] L. Flodin, V. Gandikota, and A. Mazumdar, "Superset technique for approximate recovery in one-bit compressed sensing," arXiv preprint arXiv:1910.13971, 2019.

[23] P. Xiao, B. Liao, and J. Li, "One-bit compressive sensing via schurconcave function minimization," IEEE Trans. Signal Process., vol. 67, no. 16 , pp. 4139-4151, 2019.

[24] S. Khobahi and M. Soltanalian, "Model-based deep learning for one-bit compressive sensing," IEEE Trans. Signal Process., vol. 68, pp. 52925307,2020 
[25] J. N. Laska, Z. Wen, W. Yin, and R. G. Baraniuk, "Trust, but verify: Fast and accurate signal recovery from 1-bit compressive measurements," IEEE Trans. Signal Process., vol. 59, no. 11, pp. 5289-5301, 2011.

[26] D. Needell and J. A. Tropp, "CoSaMP: Iterative signal recovery from incomplete and inaccurate samples," Appl. Comput. Harmon. Anal., vol. 26, no. 3, pp. 301-321, 2009.

[27] P. T. Boufounos, "Greedy sparse signal reconstruction from sign measurements," in Conf. Rec. Asilomar Conf. Signals Syst. Comput. IEEE, 2009 , pp. $1305-1309$.

[28] L. Jacques, J. N. Laska, P. T. Boufounos, and R. G. Baraniuk, "Robust 1bit compressive sensing via binary stable embeddings of sparse vectors," IEEE Trans. Inf. Theory, vol. 59, no. 4, pp. 2082-2102, 2013.

[29] M. P. Friedlander, H. Jeong, Y. Plan, and O. Yilmaz, "NBIHT: An efficient algorithm for 1-bit compressed sensing with optimal error decay rate," arXiv preprint arXiv:2012.12886, 2020.

[30] Y. Plan and R. Vershynin, "Robust 1-bit compressed sensing and sparse logistic regression: A convex programming approach," IEEE Trans. Inf. Theory, vol. 59, no. 1, pp. 482-494, 2012.

[31] L. Zhang, J. Yi, and R. Jin, "Efficient algorithms for robust one-bit compressive sensing," in Int. Conf. Mach. Learn. PMLR, 2014, pp. $820-828$.

[32] X. Cai, Z. Zhang, H. Zhang, and C. Li, "Soft consistency reconstruction: a robust 1-bit compressive sensing algorithm," in IEEE Int. Conf. Commun. IEEE, 2014, pp. 4530-4535.

[33] X. Huang, L. Shi, M. Yan, and J. A. Suykens, "Pinball loss minimization for one-bit compressive sensing: Convex models and algorithms," Neurocomputing, vol. 314, pp. 275-283, 2018.

[34] L. Rencker, F. Bach, W. Wang, and M. D. Plumbley, "Sparse recovery and dictionary learning from nonlinear compressive measurements," IEEE Trans. Signal Process., vol. 67, no. 21, pp. 5659-5670, 2019.

[35] X. Huang and M. Yan, "Nonconvex penalties with analytical solutions for one-bit compressive sensing," Signal Process., vol. 144, pp. 341-351, 2018.

[36] W. Xu, Y. Tian, S. Wang, and Y. Cui, "Feature selection and classification of noisy proteomics mass spectrometry data based on onebit perturbed compressed sensing," Bioinformatics, vol. 36, no. 16, pp. $4423-4431,2020$

[37] X. Fu, F.-M. Han, and H. Zou, "Robust 1-bit compressive sensing against sign flips," in Glob. Commun. Conf. IEEE, 2014, pp. 3121-3125.

[38] F. Li, J. Fang, H. Li, and L. Huang, "Robust one-bit bayesian compressed sensing with sign-flip errors," IEEE Signal Process. Lett., vol. 22, no. 7, pp. 857-861, 2014

[39] H.-J. M. Shi, M. Case, X. Gu, S. Tu, and D. Needell, "Methods for quantized compressed sensing," in Inf. Theory Appl. Workshop. IEEE, 2016, pp. 1-9.

[40] J. Huang, Y. Jiao, X. Lu, and L. Zhu, "Robust decoding from 1bit compressive sampling with ordinary and regularized least squares," SIAM J. Sci. Comput., vol. 40, no. 4, pp. A2062-A2086, 2018.

[41] Q. Fan, C. Jia, J. Liu, and Y. Luo, "Robust recovery in 1-bit compressive sensing via $l_{q}$-constrained least squares," Signal Process., vol. 179, p. 107822, 2021

[42] M. Yan, Y. Yang, and S. Osher, "Robust 1-bit compressive sensing using adaptive outlier pursuit," IEEE Trans. Signal Process., vol. 60, no. 7, pp. 3868-3875, 2012.

[43] A. Movahed, A. Panahi, and G. Durisi, "A robust rfpi-based 1-bit compressive sensing reconstruction algorithm," in IEEE Inf. Theory Workshop. IEEE, 2012, pp. 567-571.

[44] A. Movahed, A. Panahi, and M. C. Reed, "Recovering signals with variable sparsity levels from the noisy 1-bit compressive measurements," in IEEE Int. Conf. Acoust. Speech Signal Process. IEEE, 2014, pp. 6454-6458.

[45] D.-Q. Dai, L. Shen, Y. Xu, and N. Zhang, "Noisy 1-bit compressive sensing: models and algorithms," Appl. Comput. Harmon. Anal., vol. 40, no. 1, pp. 1-32, 2016.

[46] L. Armijo, "Minimization of functions having lipschitz continuous first partial derivatives," Pac. J. Math., vol. 16, no. 1, pp. 1-3, 1966.

[47] C. Gianelli, L. Xu, J. Li, and P. Stoica, "One-bit compressive sampling with time-varying thresholds: Maximum likelihood and the cramér-rao bound," in 50th Asilomar Conf. Signals, Syst. Comput. IEEE, 2016, pp. $399-403$.

[48] H. Zhu, F. Liu, and J. Li, "Computationally efficient sinusoidal parameter estimation from signed measurements: ADMM approaches," IEEE Signal Process. Lett., vol. 26, no. 12, pp. 1798-1802, 2019.

[49] J. Ren, T. Zhang, J. Li, and P. Stoica, "Sinusoidal parameter estimation from signed measurements via majorization-minimization based RELAX," IEEE Trans. Signal Process., vol. 67, no. 8, pp. 2173-2186, 2019.
[50] A. Beck and Y. C. Eldar, "Sparsity constrained nonlinear optimization: Optimality conditions and algorithms," SIAM J. Optim., vol. 23, no. 3, pp. 1480-1509, 2013.

[51] J. J. Moré and D. C. Sorensen, "Computing a trust region step," SIAM J. Sci. Statist. Comput., vol. 4, no. 3, pp. 553-572, 1983. 